Die Finanzierung der NRO in der

Entwicklungszusammenarbeit und der humanitären Hilfe: Der Fall der Schweiz und internationale Vergleiche

\title{
Gérard Perroulaz
}

\section{(2) OpenEdition}

\section{Journals}

Electronic version

URL: http://journals.openedition.org/sjep/498

DOI: $10.4000 /$ sjep.498

ISSN: 1663-9677

\section{Publisher}

Institut de hautes études internationales et du développement

\section{Printed version}

Date of publication: 1 novembre 2004

Number of pages: $51-86$

ISBN: 2-88247-057-6

ISSN: $1660-5926$

\section{Electronic reference}

Gérard Perroulaz, « Die Finanzierung der NRO in der Entwicklungszusammenarbeit und der

humanitären Hilfe: Der Fall der Schweiz und internationale Vergleiche », Schweizerisches Jahrbuch für Entwicklungspolitik [Online], 23-2 | 2004, Online erschienen am: 10 Juni 2010, abgerufen am 08 September 2020. URL : http://journals.openedition.org/sjep/498 ; DOI : https://doi.org/10.4000/sjep. 498 


\title{
Die Finanzierung der NRO in der Entwicklungs- zusammenarbeit und der humanitären Hilfe: Der Fall der Schweiz und internationale Vergleiche
}

\author{
Gérard Perroulaz*
}

\section{Einleitung}

Die Nichtregierungsorganisationen (NRO) spielen eine wichtige Rolle in der Entwicklungszusammenarbeit und der humanitären Hilfe der Schweiz, indem sie ihre eigenen Programme umsetzen und Programme der staatlichen Entwicklungszusammenarbeit im Auftrag durchführen. Die NRO nehmen auch eine bedeutende Stellung bei der Information der Öffentlichkeit über Entwicklungsprobleme ein, und ferner, indem sie die schweizerische Aussenpolitik gegenüber den Entwicklungsländern in kritischer Weise analysieren, wobei sie namentlich auf die mangelnde Kohärenz zwischen einer auf Solidarität beruhenden Politik und gewissen kurzfristigen Wirtschaftsinteressen hinweisen.

Im vorliegenden Artikel, der sich vor allem mit der Finanzierung der oben genannten NRO befasst, präsentieren wir die verfügbaren Daten über die Organisationen, welche vor Ort im Bereich der Entwicklungszusammenarbeit und der humanitären Hilfe tätig sind. Die statistischen Daten über diese NRO beruhen hauptsächlich auf den Angaben, die vom Universitätsinstitut für Entwicklungsstudien (iuéd) seit Anfang der 90er Jahre zusammengetragen wurden, sowie auf den von der Direktion für Entwicklung und Zusammenarbeit (DEZA) herausgebrachten Daten über die öffentlichen Beiträge an die NRO ${ }^{1}$. Die Analyse betreffend die Finanzierung der Aktivitäten der schweizerischen NRO verweist zum einen auf den Spendenmarkt in der Schweiz und zum anderen auf die Abhängigkeit der NRO gegenüber der Finanzierung durch die öffentliche Hand (DEZA, Kantone und Gemeinden). Ein internationaler Vergleich ermöglicht das Aufzeigen der Unterschiede und Ähnlichkeiten zwischen der NRO-Szene in der Schweiz und den Nichtregierungsorganisationen in anderen Mitgliedsländern des OECD-Entwicklungshilfeausschusses (DAC).

Im Jahr 2002 belief sich die gesamte Hilfe der Schweiz (öffentliche und private Leistungen) an die Entwicklungs- und Transitionsländer auf 1878 Millionen Franken. Die Hilfe des Bundes erreichte 1536 Millionen Franken, das entsprach 81,8 Prozent der gesamten Leistungen, und die Hilfe der Kantone und Gemeinden 28 Millionen Franken, das entsprach 1,5 Prozent der gesamten Leistungen. Die in

* Forschungsbeauftragter am iuéd.

1 Anhang I gibt genauere Informationen über die in der Schweiz bestehenden Daten und über die wichtigsten Quellen. Die erhältlichen Daten betreffen hauptsächlich die von den NRO zusammengetragenen privaten Mittel und die öffentlichen Finanzierungen der NRO (Bund, Kantone und Gemeinden). Es existiert keine gesamtheitliche Statistik über andere Finanzierungsquellen (private Stiftungen, ausländische öffentliche Finanzierungen, Europäische Union, internationale Organisationen). 
den Entwicklungs- oder Transitionsländern aufgewandte und durch Eigenmittel finanzierte Hilfe der NRO belief sich auf 313,8 Millionen Franken; sie macht somit 16,7 Prozent der gesamten öffentlichen und privaten Hilfe der Schweiz aus.

Die Nichtregierungsorganisationen werden finanziert durch Eigenmittel (Ertrag von Sammelkampagnen, Beiträge und Spenden der Mitglieder, Erlös aus dem Verkauf verschiedener Artikel), durch die Zahlungen anderer nationaler NRO (Glückskette oder sonstige Organisationen), durch öffentliche schweizerische Beiträge (Bund, Kantone oder Gemeinden), durch ausländische private Beiträge (z.B. eine andere nationale Zweigstelle einer internationalen NRO), durch ausländische öffentliche Beiträge (Entwicklungshilfeagentur eines anderen DACLandes) und durch die Europäische Union oder internationale Organisationen.

\section{Private Finanzierung: die NRO und der Spendenmarkt in der Schweiz}

Die vom iuéd bei den Nichtregierungsorganisationen seit 1991 durchgeführten Jahresumfragen haben ergeben, dass über 600 schweizerische NRO in der Verwaltung von Programmen und Projekten in Entwicklungs- oder Transitionsländern tätig sind. Anhand der Antworten auf diese Umfragen lässt sich die obige Schätzung des Gesamtbetrags der Hilfe der NRO aus privaten Mitteln vornehmen, der sich im Jahr 2002 auf 313,8 Millionen Franken belief. Dieser Betrag entspricht nicht der Gesamtsumme der Budgets der betreffenden NRO, sondern lediglich dem Betrag, der in Entwicklungs- und Transitionsländern für Projekte ausgegeben wird, welche mit Eigenmitteln der NRO finanziert werden. Er schliesst weder die Beiträge des öffentlichen Sektors, noch die Arbeit der NRO im Bereich der Sensibilisierung für Entwicklungsfragen, noch die Verwaltungsausgaben in der Schweiz mit ein.

Die Beträge der privaten Finanzierung von NRO-Projekten müssen in den Kontext des so genannten „Spendenmarktes“ gestellt werden. Die Zentralstelle für Wohlfahrtsunternehmen ZEWO $^{2}$ schätzt, dass sich das Volumen der in der schweizerischen Bevölkerung gesammelten Spenden im Jahr 2002 für die rund 300 ZEWO zertifizierten Organisationen auf 610 Millionen Franken belief. Diese privaten Spenden der NRO setzen sich aus Einzelspenden (2002 : 290 Millionen Franken), Mitgliederbeiträgen (100 Millionen Franken, von Jahr zu Jahr in etwa gleichbleibender Betrag), institutionellen Spenden (90 Millionen Franken an Beiträgen von Stiftungen, Vereinen oder Kirchen), Legaten (90 Millionen Franken) und sonstigen Spenden (40 Millionen Franken) zusammen. Die Spendeneinnahmen sind von 2001 auf 2002 deutlich zurückgegangen, indem sie von 700 Millionen auf 610 Millionen Franken abfielen. Dies ist insbesondere auf das aussergewöhnliche Spendenaufkommen im Jahr 2001 nach dem Unwetter in Gondo (Wallis) zurückzuführen.

2 Siehe den Artikel von Ariuscha Davatz in diesem Dossier: Das ZEWO-Gütesiegel für gemeinnützige NRO als Beitrag zu erhöhter Transparenz auf dem Schweizer Spendenmarkt. Die ZEWO schätzt, dass sich die Gesamtfinanzierungen der von ihnen zertifizierten NRO im Jahr 2002 auf 1,7 Milliarden Franken beliefen. Die Haupteinnahmen sind die privaten Spenden (36\% der Gesamteinnahmen) und die öffentlichen Beiträge (580 Millionen Franken, d.h. 34\% des Gesamtbetrags), Eigenleistungen (Erträge aus Verkäufen und Aufträgen, $22 \%$ ) und sonstige Einnahmen ( $8 \%$ ). 


\section{Entwicklung der privaten Finanzierung der NRO}

Grafik 1 zeigt die Entwicklung der aus Eigenmitteln finanzierten Hilfe der NRO (ohne öffentliche Beiträge von DEZA, Kantonen und Gemeinden) über einen Zeitraum von rund zwanzig Jahren. Die Leistungen der NRO haben in dieser Periode ziemlich regelmässig zugenommen ${ }^{3}$.

\section{Grafik 1: Entwicklung der aus Eigenmitteln finanzierten Hilfe der NRO, 1985-2002 (in Millionen Franken)}

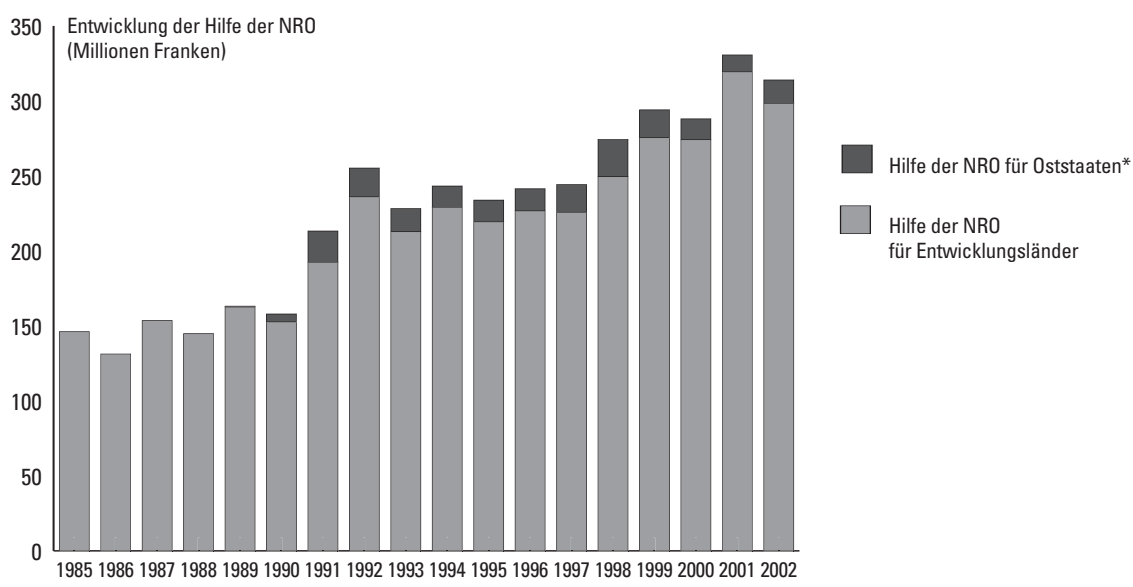

* Hilfe der NRO für Oststaaten: Hilfe für Transitionsländer (Länder Mittel- und Osteuropas und fortgeschrittenere Entwicklungsländer, 2. Teil der DAC-Liste). Die DAC-Liste der Hilfeempfängerländer wird jedes Jahr vom Entwicklungshilfeausschuss der OECD (DAC) festgelegt. Quellen: Jahresumfragen über die Hilfe der NRO, im Auftrag der DEZA bis 1990 von Swissaid und seit 1991 vom iuéd durchgeführt.

Bei der Analyse der Entwicklung der mit Eigenmitteln der Organisationen finanzierten Hilfe ist Vorsicht geboten. Die Schwankungen der privaten Hilfe von einem Jahr aufs andere können auch durch statistische Faktoren beeinflusst werden, zum Beispiel durch die Zunahme der Anzahl NRO, welche die jährlichen Fragebögen beantworten (1990 : 130 Organisationen, 2002 : rund 250 Organisationen). In der Tat sind die NRO nicht gehalten, die statistischen Erhebungen zu beantworten. Die Tatsache, dass eine grosse NRO ihre Mittel in einem Jahr angibt und im darauf folgenden Jahr nicht deklariert, kann den Gesamtbetrag der Hilfe absinken lassen, ohne dass dies eine allgemein rückläufige Tendenz anzeigt.

\section{Je nach den Organisationen sehr unterschiedliche Entwicklung}

Die Entwicklung der privaten Finanzierung der NRO ist natürlich von einer Organisation zur anderen unterschiedlich. Anhand der Datenbank des iuéd über die Hilfe von zirka 200 Nichtregierungsorganisationen von 1990 bis 2002 was jedes Jahr rund 95 bis 98 Prozent der gesamten privaten Hilfe entspricht lassen sich folgende Merkmale und Tendenzen herausstellen: 
- Einige grosse oder auch kleine NRO haben eine relativ stabile Entwicklung ihrer Tätigkeit verzeichnet. Unter ihnen kann man Terre des Hommes Lausanne nennen (Änderungen von Jahr zu Jahr, jedoch ziemlich beständige langfristige Entwicklung, mit einem Projektgesamtbetrag, der jährlich zwischen 12 und 18 Millionen Franken schwankt). Die Entwicklung der privaten Projektfinanzierung ist beispielsweise auch für die Organisationen Département Missionnaire, Frères de nos frères und Kinderdirekthilfe Kolumbien recht stabil.

- Andere NRO verzeichneten einen starken Aufschwung ihrer Tätigkeit. Das Schweizerische Komitee für UNICEF konnte zum Beispiel sein Mittelaufkommen mit rund 22 Millionen Franken für die Finanzierung von Projekten im Jahr 2002 (gegenüber 12 Millionen 1991) beträchtlich erhöhen.

- Der Spendenmarkt ist durch das Auftreten neuer NRO gekennzeichnet, von denen einige beim Spendensammeln bei der schweizerischen Bevölkerung eine bedeutende Stellung erlangt haben. Dies ist bei grossen internationalen Nichtregierungsorganisationen der Fall, die sich allmählich auf dem schweizerischen Markt niedergelassen haben. Hierzu sei beispielsweise der starke Aufschwung der Organisation Ärzte ohne Grenzen genannt, deren Finanzierung durch Eigenmittel von 1,1 Millionen Franken 1990 auf 10 Millionen 1998 und auf 23 Millionen im Jahr 2001 angestiegen ist ${ }^{4}$. Ein weiteres Beispiel ist World Vision, deren Eigenmittelfinanzierung sich von 1,9 Millionen Franken 1991 auf 15,3 Millionen im Jahr 2002 erhöhte.

Anhand dieser Feststellungen kann man sich fragen, ob das Aufkommen neuer nichtstaatlicher Organisationen oder die starke Zunahme der Tätigkeit einiger bestehender Organisationen auf Kosten der grossen traditionellen NRO erfolgte 5 . Die Antwort darauf ist nuanciert, trotz der alarmierenden Artikel, welche regelmässig in der Schweiz erscheinen, wenn einige NRO sich über den Rückgang ihres Spendenaufkommens beunruhigen. Man kann eine deutlich rückläufige Tendenz der Spendeneinnahmen bei gewissen NRO feststellen, wie zum Beispiel Leprahilfe Emmaus und Association suisse d'aide à l'hôpital Albert Schweitzer.

Andere herkömmliche Organisationen verzeichnen von einem Jahr aufs andere sehr starke Schwankungen, namentlich in Bezug auf den Umfang ihrer humanitären Hilfe. Dies ist beim Schweizerischen Roten Kreuz, bei Caritas Schweiz und beim Schweizerischen Arbeiterhilfswerk der Fall.

Einige traditionelle NRO erfuhren hingegen einen Aufschwung ihrer Tätigkeit. Die durch Eigenmittel finanzierten Aktivitäten von Swissaid verzeichneten eine steigende Tendenz, mit Projekten im Wert von 870’000 Franken 1991 und 3,2 Millionen Franken im Jahr 2002. Der gleiche Trend lässt sich bei Swisscontact (Steigerung der Leistungen von 1,3 Millionen Franken 1991 auf 2,2 Millionen 2002) und bei Helvetas (Zunahme von 3,4 Millionen Franken 1991 auf 5,8 MiIlionen 2002) feststellen. Auch bei der Stiftung Kinderdorf Pestalozzi ist eine steigende Tendenz zu beobachten.

4 Hingegen hat die Organisation Médecins du Monde, trotz ihrer Bedeutung in Frankreich, in der Schweiz nicht sehr stark Fuss gefasst.

5 Wir betrachten als ,traditionelle“ Organisationen die seit den 50er Jahren bis Ende der 70er Jahre gegründeten Hilfsorganisationen, wie zum Beispiel Helvetas, Swisscontact, HEKS, Schweizerisches Arbeiterhilfswerk, Caritas und Terre des Hommes. 
Somit haben sich die auf dem „Spendenmarkt" neu aufgetretenen Organisationen vor allem ein Stück aus einem Kuchen geholt, der grösser wird, und ihr Aufschwung erfolgte nicht zwangsläufig auf Kosten traditionellerer Organisationen.

Unter den kleinen NRO haben einige nur eine beschränkte Lebensdauer: sie treten plötzlich in Erscheinung, um dann nach ein paar Jahren wieder zu verschwinden oder an Bedeutung zu verlieren. Das Beispiel jener NRO, deren Tätigkeit mit der Krise in Rumänien verbunden war, ist in dieser Hinsicht frappant. Man kann dieses Phänomen als positiv betrachten (rasche Mobilisierung, um einem Land oder einem Gebiet punktuell während einer schwierigen Phase zu helfen), oder auch mit Beunruhigung verfolgen (Modeerscheinung mit sehr kurzfristigen Aktionen). Viele NRO, die sich mit der Hilfe für die Oststaaten beschäftigt hatten, waren kurzlebig (z.B. Pro Transsylvania oder Kroatisches Humanitäres Forum).

\section{Bedeutung der den Kirchen nahe stehenden Organisationen}

Die Missionswerke spielen beim Spendensammeln in der schweizerischen Bevölkerung eine sehr wichtige Rolle. Die ersten humanitären Einsätze der Schweiz gehen ins 19. Jahrhundert zurück, mit der Präsenz von Missionaren in einigen Ländern des Südens (zum Beispiel Indien), in denen die Schweiz zuerst durch ihre Missionare und ihre Unternehmen auftrat. Es wurden zahlreiche Evangelisierungs- und christliche Wohltätigkeitsaktionen (Taufe armer Waisen ${ }^{6}$, Hilfe im Gesundheits- und Bildungsbereich, Unterstützung von Waisenhäusern) durchgeführt. Für die Missionswerke werden in der Hilfestatistik nur Zahlungen verbucht, die der Definition der Entwicklungszusammenarbeit und der humanitären Hilfe entsprechen?

Man könnte meinen, dass die mit den Kirchen verbundenen Organisationen mit der Zeit und mit dem Rückgang religiöser Praxis deutlich an Bedeutung verlieren würden. Jedoch zeigen die Zahlen über die Hilfe der NRO, dass dies nicht der Fall ist. Von den 250 Organisationen, bei denen man die Liste der geförderten Projekte kennt, sind über 70 Missionsorganisationen im engeren Sinne oder den Kirchen nahe stehende Institutionen. Der Anteil der von diesen Organisationen für Projekte im Süden bzw. im Osten aufgewandten Gelder ging von 71 Prozent 1980 auf 53 Prozent 1997 zurück und beläuft sich 2002 noch auf 52 Prozent der privaten Hilfe. Somit sind die über die Religionsgemeinschaften gesammelten Mittel weiterhin sehr bedeutend, und die bestehenden Statistiken unterschätzen diese $\mathrm{Hilfe}^{8}$. Tatsächlich scheinen einige nichtkonfessionelle

6 Siehe unter anderem die regelmässigen Anzeigen des Instituts für Auslandsmissionen Bethlehem Immensee in der Publikation „Echo illustré“ Anfang der 30er Jahre. Dort ist zum Beispiel am 14. März 1931 zu lesen: „Am meisten Mitleid verdienen sicherlich diese unzähligen kleinen Heidenkinder, die Gefahr laufen, ungetauft zu sterben. Ihr Seelenheil und ihr körperliches Wohl hängt von Ihnen ab. Eine Spende von 25 Franken für die christliche Erziehung eines dieser Kleinen sichert Ihnen den Segen des Göttlichen Kinderfreundes“.

7 Die Finanzierung von Missionstätigkeiten wie religiöse Publikationen, Beiträge an die Kirchen, die Bezahlung von Missionaren oder die Evangelisierungstätigkeit im engeren Sinne ist nicht in den Angaben über die Hilfe der NRO enthalten.

8 Über 70 den Kirchen nahe stehende nichtstaatliche Organisationen stellen Informationen über die Mittel bereit, die für humanitäre Hilfe und für Entwicklungsprojekte eingesetzt werden. Die Statistiken berücksichtigen die Missionsaufwendungen im engeren Sinne (Missionarsgehälter, Religions- 
Organisationen in den letzten Jahren genau so stark vom Spendenrückgang betroffen zu sein wie den Kirchen nahe stehende Organisationen".

Die den Kirchen nahe stehenden NRO, welche 2002 die höchsten Beträge an privaten Mitteln gesammelt haben, sind Caritas Schweiz (27,7 Millionen Franken), das Hilfswerk der Evangelischen Kirchen der Schweiz (HEKS) (19,5 Millionen), die Christoffel-Blindenmission (6,2 Millionen), Fastenopfer (5,6 Millionen), Bethlehem Mission Immensee (5,2 Millionen) und Mission $21^{10}(4,2$ Millionen). Danach kommen das Institut (Kloster) Baldegg, das Seraphische Liebeswerk, Christian Solidarity International, Leprahilfe Emmaus, ADRA, Brot für alle und die Limmat-Stiftung.

Man kann somit eine allgemein rückläufige Tendenz des „Spendenmarkts“ der kirchlichen Organisationen feststellen, jedoch ist die Situation je nach den Organisationen verschieden. Einige den Kirchen nahe stehende traditionelle NRO haben ihre Hilfe von 1990 bis 2002 erhöht, wie dies beispielsweise bei den Institutionen Steyler Missionare, Evangelische Nilland-Mission, ADRA, Vereinigung Don Bosco Werk oder Département Missionnaire (Echange et Mission), Limmat-Stiftung, Christoffel-Blindenmission und World Vision der Fall ist.

\section{Öffentliche Finanzierung der NRO}

Die NRO haben in der schweizerischen Entwicklungszusammenarbeit eine Vorreiterrolle gespielt, lange bevor sich der Bund in der Finanzierung von Entwicklungshilfeprojekten engagierte. Bis Ende der 40er Jahre vertrat der Bund den Standpunkt, Entwicklungshilfe sei eine Aufgabe der privaten Hilfswerke und nicht der Regierung. Ab 1950 finanziert der Bund die ersten Entwicklungsprojekte, zuerst in Nepal, mit der Entsendung von Schweizer Experten vor Ort und mit der Finanzierung von Stipendiaten aus Ländern des Südens für ihre Ausbildung in der Schweiz.

Ein Teil der Finanzierung der NRO wird von der öffentlichen Hand (Bund, aber auch Kantone und Gemeinden) bereitgestellt. Der grösste Teil der Hilfe der Schweizer Kantone und Gemeinden wird den NRO für die Durchführung von Projekten der Entwicklungszusammenarbeit ausgezahlt. Die über NRO laufenden Beiträge des Bundes können die vier folgenden Formen annehmen (Gesamtbetrag im Jahr 2002 : 209 Millionen Franken):

- Programmbeiträge der DEZA an rund 15 nichtstaatliche Organisationen (2002 : 60,2 Millionen Franken);

unterricht, Verbreitung religiöser Drucksachen) nicht, und man hat keine genauen Angaben über die von den Kirchen gesammelten Mittel, welche nicht über NRO oder über Missionsorganisationen laufen (z.B. Direktüberweisung von Mitteln von einer Schweizer Pfarrgemeinde an eine Pfarrgemeinde in Afrika, Überweisung gesammelter Mittel an einzelne Missionare).

9 Neue Luzerner Zeitung, 2. Februar 2004. In diesem Artikel stellt der Journalist einen Spendenrückgang beim Schweizerischen Roten Kreuz (Zürich), beim Hilfswerk der Evangelischen Kirchen der Schweiz, bei Mission 21 oder Fastenopfer, aber auch bei nichtkonfessionellen Organisationen wie dem Arbeiterhilfswerk oder Pro Infirmis fest.

10 Mission 21 ist das Ergebnis der Zusammenlegung folgender Organisationen im Jahr 2001 : Basler Mission, Evangelische Mission im Kwango, Mission der Brüdergemeinde und Schweizerische Ostasien-Mission (SOAM). 
\ Beiträge der DEZA für humanitäre Hilfe (Geld- oder Sachbeiträge für Nahrungsmittelhilfe, 2002 : rund 20 Millionen Franken);

- Spezifische Mandate (früher Regieprojekte genannt). Dabei handelt es sich um einen Beitrag der DEZA für von ihr festgelegte Projekte, (2002 : 123,9 Millionen Franken). Zu dieser Summe kommen noch die spezifischen Mandate des Staatssekretariats für Wirtschaft (seco) hinzu (2002 : 4 Millionen Franken). Dies ist praktisch öffentliche Hilfe für Projekte, deren Durchführung privaten Hilfswerken anvertraut wird. (Siehe Anhang II betreffend die Einzelheiten der öffentlichen Beiträge an die schweizerischen NRO.)

Grafik 2 zeigt, dass rund 29 Prozent der gesamten öffentlichen und privaten Hilfe der Schweiz von den NRO verwaltet wird, was im Jahr 2002 einen Gesamtbetrag von 549 Millionen Franken ausmacht. Diese Summe umfasst die von den Hilfswerken selbst gesammelten Beträge, das heisst 314 Millionen Franken Eigenmittel, sowie die von den öffentlichen Behörden aufgebrachten Beiträge. Ohne die spezifischen Mandate oder Regieprojekte, welche praktisch als öffentliche Hilfe angesehen werden können, belief sich die von den NRO verwaltete Hilfe im Jahr 2002 auf 420 Millionen Franken, das heisst 22,4 Prozent der gesamten öffentlichen und privaten Hilfe der Schweiz.

Grafik 2: Anteil der gesamten von den NRO verwalteten (öffentlichen und privaten) Hilfe, 2002

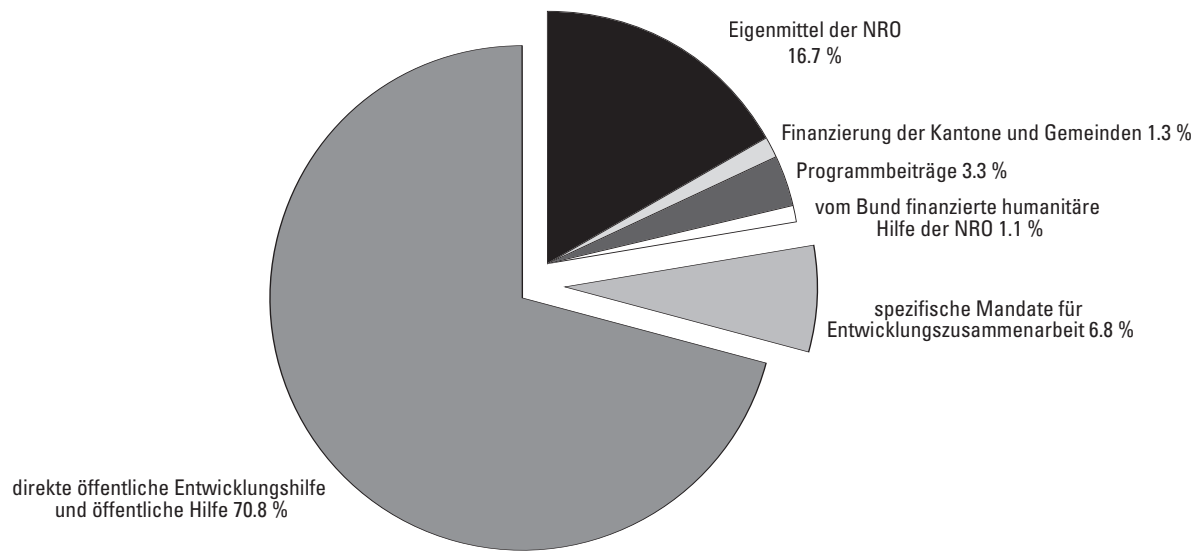

Quelle: Erstellung anhand der Broschüre der DEZA, Aide de la Suisse aux pays en développement et aux pays en transition, Statistiques 2002, Bern, DEZA, 2004, $200 \mathrm{~S}$.

Die Angaben aus Tätigkeitsberichten verschiedener Nichtregierungsorganisationen, die im Anhang III wiedergegeben sind, zeigen, dass der Anteil der öffentlichen Finanzierung der NRO von einer Organisation zur anderen äusserst verschieden ist. Institutionen wie Swisscontact und Helvetas sind von den staatlichen Mitteln stark abhängig: 79 Prozent der Einnahmen von Swisscontact werden vom Bund bereitgestellt. Hingegen macht die öffentliche Finanzierung bei Organisationen wie Ärzte ohne Grenzen weniger als 15 Prozent der Einnahmen aus.

Die umseitige Tabelle 1 vermittelt eine Vorstellung von der Entwicklung der öffentlichen Beiträge an die NRO. 


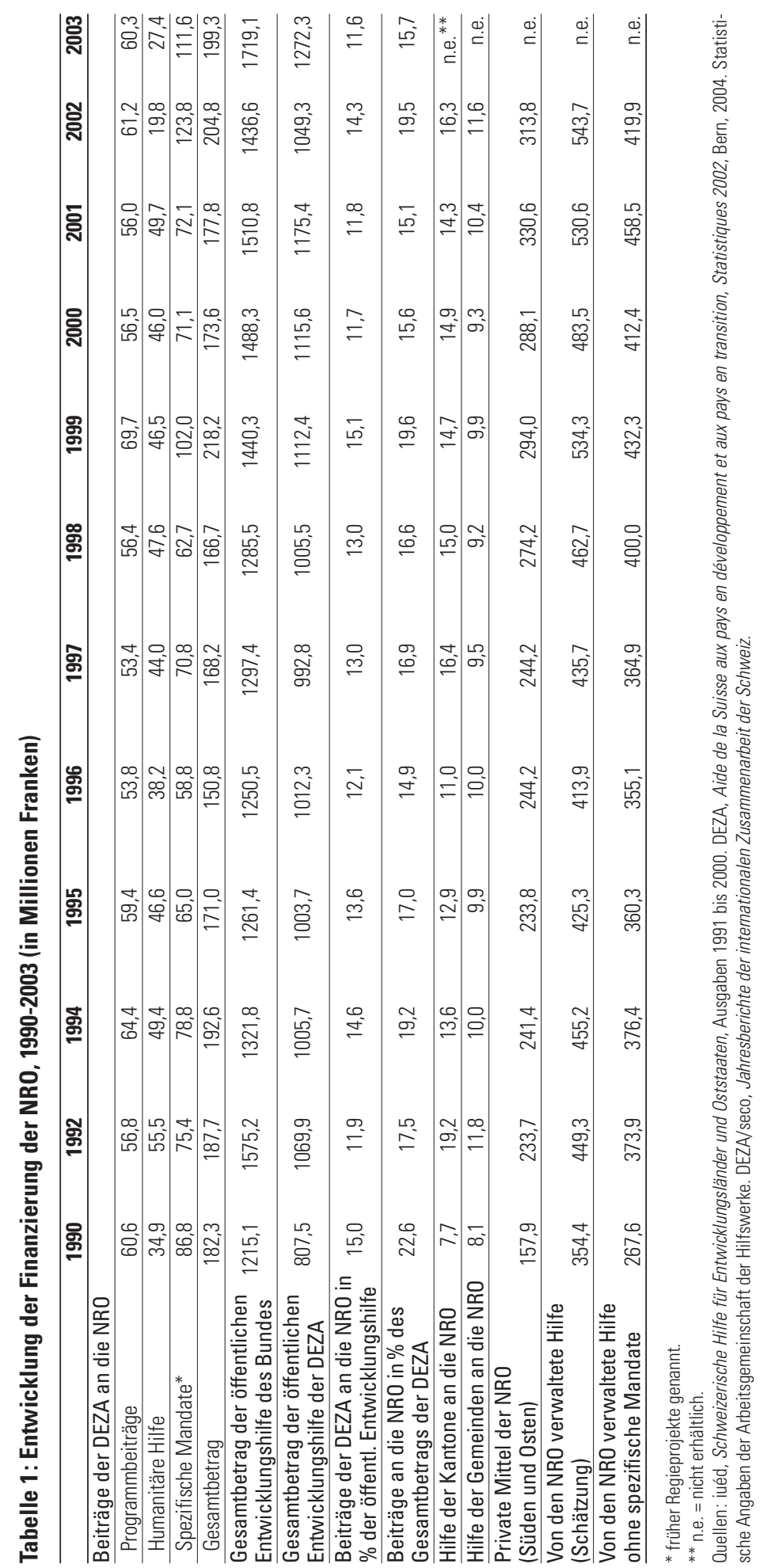


Die von den NRO verwaltete Hilfe ist in Tabelle 1 geschätzt, wobei die Beiträge der DEZA sowie 90 Prozent der Hilfe der Kantone und Gemeinden ${ }^{11}$ und die zur Projektfinanzierung eingesetzten Eigenmittel der NRO berücksichtigt werden. Die Angaben über die spezifischen Mandate der DEZA umfassen lediglich die den NRO erteilten Aufträge, ohne die Aufträge an schweizerische Unternehmen oder Beratungsbüros zu berücksichtigen, für die nicht für sämtliche Jahre Daten zur Verfügung stehen. Der Betrag der an den privaten Geschäftssektor ergangenen Aufträge war in den 80er Jahren sehr gering. Die Aufträge machten im Jahr 1992 28 Millionen Franken aus und erreichten im Jahr 200250 Millionen Franken.

Man kann feststellen, dass die Programmbeiträge der DEZA an die Hilfswerke von 1990 bis 2002 ziemlich stabil geblieben sind, wohingegen die Regieprojekte oder spezifischen Mandate deutlich anstiegen. Die Gesamtbeiträge der DEZA an die Hilfswerke haben von 1990 bis 2002 volumenmässig um 12 Prozent zugenommen, während sich die gesamte öffentliche Entwicklungshilfe der DEZA im gleichen Zeitrum um 30 Prozent erhöhte (insbesondere starke Steigerung der multilateralen Entwicklungshilfe). Die aus Eigenmitteln finanzierte Hilfe der NRO hat sich in diesem Zeitraum nahezu verdoppelt.

Eine Studie des Entwicklungszentrum der OECD erlaubt einen Vergleich der Struktur der öffentlichen Finanzierung der nichtstaatlichen Organisationen in verschiedenen Ländern ${ }^{12}$. Gemäss dieser Untersuchung ist der Anteil der öffentlichen Mittel an den finanziellen Ressourcen der NRO in den meisten Ländern hoch: 71 Prozent in Griechenland, 65 Prozent in Dänemark, 47 Prozent in Grossbritannien, 46 Prozent in Norwegen, 42 Prozent in der Schweiz, 38 Prozent in Frankreich, 32 Prozent in Deutschland, 29 Prozent in Italien und 23 Prozent in Österreich (Zahlen für 1993).

\section{Politik der DEZA gegenüber den NRO für Entwicklungszusammenarbeit'13}

Mehrere Ämter der Bundesverwaltung haben Kontakte zu schweizerischen NRO und finanzieren deren Aktivitäten, und es ist eingangs festzustellen, dass es keine gesamtheitliche Politik des Bundes gegenüber diesen Organisationen gibt. Der NGO-Dienst der DEZA ist zweifellos der wichtigste „Berührungspunkt“ der NRO mit der Bundesverwaltung, jedoch haben auch andere Bundesämter verschiedener Departemente Verbindungen zu den NRO, wie zum Beispiel die Politische Abteilung IV des Eidgenössischen Departements für auswärtige Angelegenheiten (EDA), für die Förderung von Frieden und Menschenrechten, das Eidgenössische Departement für Verteidigung, Bevölkerungsschutz und Sport (VBS), für die Friedensförderung, und das Bundesamt für Umwelt, Wald und Landschaft (BUWAL), bezüglich der Umweltpolitik. Die eine oder andere NRO unterhält privilegierte

11 Man kann schätzen, dass in der Schweiz 90\% der Hilfe der Kantone und Gemeinden zur Finanzierung von Projekten dienen, die von Hilfswerken verwaltet werden. Die direkte Hilfe, zum Beispiel im Rahmen von Gemeindepartnerschaften, ist in der Schweiz nicht bedeutend.

12 Adèle Woods, Les ONG européennes actives dans le domaine du développement. Etat des lieux, Etudes du Centre de Développement, Paris, OECD, 2000, 96 S.

13 Zur Politik der DEZA gegenüber den entwicklungspolitischen NRO siehe auch den Artikel von Bundesrat Joseph Deiss, Vorsteher des Eidgenössischen Volkswirtschaftsdepartements, und Walter Fust, Direktor der DEZA, in diesem Dossier sowie das Interview mit Walter Fust, welches über die Zukunft der Beziehungen zwischen der DEZA und den NRO informiert. Ferner gibt die Tabelle im Anhang I dieses Artikels einen Überblick über die Beiträge des Bundes an rund hundert NRO im Jahr 2002. 
Beziehungen zum einen oder anderen Bundesamt. Eine im vorliegenden Dossier erwähnte neue Studie über die Rolle des internationalen Genfs weist auch auf die Schwächen bei der Koordination zwischen den verschiedenen Ämtern der Eidgenossenschaft und den Genfer Kantonalbehörden bezüglich ihrer jeweiligen Politik gegenüber den internationalen NRO in Genf hin ${ }^{14}$.

Die Politik der DEZA gegenüber den Nichtregierungsorganisationen ist in mehreren offiziellen Dokumenten festgehalten, insbesondere in der letzten Botschaft des Bundesrates über die Weiterführung der technischen Zusammenarbeit ${ }^{15}$. Für den Bundesrat sind die NRO ,wertvolle Partner zur Führung eines intensiven und lebhaften entwicklungspolitischen Dialoges und wichtige Partner für die Umsetzung von Projekten und Programmen der DEZA“. „Dialog und Erfahrungsaustausch sowie die gegenseitige Auseinandersetzung über Themen von gemeinsamem Interesse zwischen der DEZA und den NGOs dienen der Förderung von Komplementaritäten. Die NGOs leisten einen Beitrag zu einer lebendigen entwicklungspolitischen Diskussion und können innovative Impulse zur qualitativen Evolution der schweizerischen Entwicklungszusammenarbeit schaffen" ${ }^{16}$. Die DEZA legt Nachdruck auf die Komplementarität der Rollen und jeweiligen komparativen Vorteile der staatlichen Akteure, der schweizerischen NRO und der Zivilgesellschaft in den Ländern des Südens. Die NRO und ihre Partner im Süden verfügen manchmal über Handlungsmöglichkeiten, welche staatlichen Stellen verwehrt sind. Im Jahr 2004 ist eine Evaluation im Gang, die der DEZA eine klarere Ausrichtung und bessere Koordination ihrer Politik gegenüber den NRO erlauben soll.

Die Zusammenarbeit zwischen dem Staat und den privaten Hilfswerken bildet einen Schwerpunkt der Analyse der Rolle der NRO in der Entwicklungszusammenarbeit; eine umfangreiche Literatur befasst sich mit diesem Thema. Die Tendenz der letzten beiden Jahrzehnte lag bei einer Stärkung der Beziehungen zwischen öffentlicher Hand und NRO.

Für die Schweiz identifiziert Richard Gerster sechs Funktionen der NRO gegenüber dem öffentlichen Sektor ${ }^{17}$ :

- Nutzniessende Funktion. Die NRO werden bei ihrer Tätigkeit durch Programmbeiträge der DEZA finanziell unterstützt, die im Allgemeinen 50 Prozent der Projektkosten ausmachen; den Rest finanzieren die NRO aus ihren Eigenmitteln.

- Exekutive Funktion. Die NRO führen von der DEZA festgelegte Projekte auf der Grundlage spezifischer Mandate durch (früher „Regieprojekte“).

- Funktion zur Unterstützung der Entwicklungspolitik durch Informationsarbeit, Sensibilisierung der Öffentlichkeit und entwicklungspolitische Bildung. Diese Arbeit ist für die Regierung natürlich wertvoll, da sie die Öffentlichkeit

14 Siehe den Artikel von Yanik Marguerat in diesem Dossier: Die internationalen NRO in Genf.

15 Botschaft über die Weiterführung der technischen Zusammenarbeit und der Finanzhilfe zu Gunsten von Entwicklungsländern, vom 28. Mai 2003, 4.4.2., „Die Zusammenarbeit mit der Zivilgesellschaft sowie öffentlichen Körperschaften der Kantone“, Bundesblatt 2003, S. 4667-4472.

16 Botschaft über die Weiterführung der technischen Zusammenarbeit, op. cit, S. 4668.

17 Richard Gerster, „Die Zusammenarbeit zwischen dem Staat und privaten Hilfswerken. Erfahrungen aus der schweizerischen Entwicklungspolitik" in Helmich, Henny; Bittner, Gerhard, (Hrsg.), Der dritte Sektor in der Entwicklungspolitik. Zusammenarbeit zwischen Staat und Nicht-Regierungsorganisationen - NROs. Wien, Österreichische Forschungsstiftung für Entwicklungshilfe, 1996, S. 135-145. 
für die Bedeutung der Entwicklungszusammenarbeit aufgeschlossener macht. Die NRO sind klar die Verbündeten der DEZA, wenn sie zum Beispiel eine Erhöhung der öffentlichen Entwicklungshilfe fordern.

- Katalytische Funktion. Ein wichtiges Beispiel hierzu ist die von den NRO Anfang der 90er Jahre lancierte Petition „Entwicklung braucht Entschuldung“, welche 250'000 Unterschriften einbrachte. Diese Petition mündete in einen öffentlichen Rahmenkredit von 700 Millionen Franken, der für Entschuldungsmassnahmen und Initiativen im Umweltbereich bestimmt war. Ferner war der Beitrag der Hilfswerke bei der Erarbeitung des Bundesgesetzes über die Entwicklungszusammenarbeit und die humanitäre Hilfe von 1976 massgeblich.

- Kritische Funktion zum Anprangern gewisser Inkohärenzen in der schweizerischen Aussenpolitik.

- Beratende Funktion. Die Arbeitsgemeinschaft der Hilfswerke ist zu einem privilegierten Gesprächspartner der DEZA und des seco geworden (Verwaltung der Gegenwertfonds im Entschuldungsbereich) und sie erhält regelmässig Mandate für gewisse Studien. Vertreter der Hilfswerke haben in einigen ausserparlamentarischen Kommissionen Einsitz und sind in schweizerischen Delegationen bei internationalen Konferenzen integriert.

\section{Der Spendenmarkt für die Entwicklungszusammenarbeit} und die humanitäre Hilfe

Anhand der Analyse der Zahlenangaben zur Finanzierung der NRO lassen sich einige Merkmale des Spendenmarkts in der Schweiz im Bereich der Entwicklungszusammenarbeit und der humanitären Hilfe identifizieren:

\section{Wenige grosse Institutionen vereinen die Hälfte der privaten finanziellen Mittel auf sich}

Tabelle 2 zeigt, dass die neun grössten Nichtregierungsorganisationen im Jahr 200251 Prozent der gesamten Eigenmittel der NRO ausmachen ${ }^{18}$. Die 38 grössten NRO, mit Eigenmitteln von über 2 Millionen Franken, machen über 80 Prozent der gesamten von den NRO zusammengetragenen Mittel aus. Am anderen Ende verwalten die 159 kleinsten Organisationen lediglich 10 Prozent der für die Hilfe für den Süden gesammelten Gelder. Diese Unterschiede werfen auch Licht auf die Finanzmittel, die investiert werden können, um neue Geldgeber anzuziehen. Es ist in der Tat einfacher, beispielsweise Kampagnen mit Plakatanschlägen und Presseanzeigen zu finanzieren, wenn es sich um eine Organisation mit einem Gesamthaushalt von mehreren Millionen Franken handelt, als wenn es um eine kleine Organisation geht, die nur über ein Jahresbudget von insgesamt 100 '000 Franken verfügt ${ }^{19}$.

18 Die neun grössten NRO in Bezug auf die private Finanzierung im Jahr 2002 sind (in absteigender Reichenfolge): Caritas, das Schweizerische Komitee für UNICEF, das Hilfswerk der Evangelischen Kirchen der Schweiz, die Stiftung Kinderspital Kantha Bopha, Ärzte ohne Grenzen, World Vision, Terre des Hommes Lausanne, das Schweizerische Rote Kreuz und Schweizer Freunde der SOS-Kinderdörfer.

19 Im Anhang II sind die Angaben für an die hundert nichtstaatliche Organisationen aufgeführt, welche rund $95 \%$ des Gesamtbetrags der Hilfe der schweizerischen NRO ausmachen, mit Informationen über die Aktionen der durch öffentliche Beiträge finanzierten NRO. 
Tabelle 2: Aufteilung der NRO nach der Höhe ihrer Eigenmittel, 2002 (in Tausend Franken)

\begin{tabular}{lcccr}
\hline Eigenmittel der NRO & AnzahI NRO & Tausend Franken & $\begin{array}{c}\text { in \% des } \\
\text { Gesamtbetrags }\end{array}$ & $\begin{array}{r}\text { in \% } \\
\text { kumuliert }\end{array}$ \\
\hline über 20 Millionen Franken & 2 & 49605 & 15.8 & \\
\hline zwischen 10 und 20 Millionen & 7 & 111183 & 35.4 & 51.2 \\
\hline zwischen 5 und 10 Millionen & 5 & 27975 & 8.9 & 60.1 \\
\hline zwischen 2 und 5 Millionen Franken & 24 & 64878 & 20.7 & 80.8 \\
\hline 1 bis 2 Millionen Franken & 20 & 27643 & 8.8 & 89.6 \\
\hline unter 1 Million Franken & 159 & 32559 & 10.4 & 100.0 \\
\hline Gesamtbetrag & $\mathbf{2 1 7}$ & $\mathbf{3 1 3 8 4 3}$ & $\mathbf{1 0 0 . 0}$ & \\
\hline
\end{tabular}

Quelle: Berechnung anhand der Angaben der Broschüre Aide de la Suisse aux pays en développement et aux pays en transition, Statistiques 2002, Bern, DEZA, 2004.

Diese Konzentration der Entwicklungshilfe auf einige grosse NRO ist keine schweizerische Ausnahme. Die gleiche Situation herrscht in den meisten Ländern des Nordens vor. Gemäss der oben erwähnten OECD-Studie mit Daten für 1993 verwalten in mehreren europäischen Ländern 20 Prozent der grössten nichtstaatlichen Organisationen über 86 Prozent der Ressourcen der gesamten NRO des Landes. Dies ist beispielsweise in Deutschland, Österreich, Dänemark, Spanien, Frankreich, Italien, den Niederlanden, Grossbritannien, Schweden und der Schweiz der Fall (mit einem Durchschnitt von rund 90\% der Mittel für die genannten Länder $)^{20}$.

Die Finanzmittel der wichtigsten europäischen NRO sind sehr bedeutend, und die Funktionsweise dieser Organisationen ist weit entfernt vom kleinen Verein mit einem engagierten Präsidenten. Laut einer französischen Studie von 1999 beliefen sich die Budgets der grössten europäischen NRO beispielsweise auf 196 Millionen Euro für Oxfam (Grossbritannien), 157 Millionen Euro für Misereor (Deutschland), 154 Millionen Euro für Save the Children (Grossbritannien), 150 Millionen Euro für Novib (Niederlande), 136 Millionen für Cordaid (Niederlande) sowie 94 Millionen Euro für den Dienst der Evangelischen Kirchen in Deutschland ${ }^{21}$.

In der Schweiz belief sich das Gesamtbudget von Caritas Schweiz im Jahr 2003 auf 103,2 Millionen Franken (einschliesslich der sozialen Aktion und der Unterstützung für die Flüchtlinge in der Schweiz); das Budget des Schweizerischen Arbeiterhilfswerks betrug 56,6 Millionen Franken, dasjenige von Helvetas 53,2 Millionen Franken, von Ärzte ohne Grenzen Schweiz 52,6 Millionen Franken und von World Vision 26,9 Millionen Franken.

\section{Scharfe Konkurrenz unter den NRO auf dem Spendenmarkt}

Die oben aufgeführte, insgesamt positive Entwicklung der privaten Spenden, die ziemlich regelmässige Zunahme der privaten Mittel der schweizerischen NRO, verbirgt jedoch gewisse Probleme. Der Wettbewerb unter den NRO auf dem Spendenmarkt ist sehr gross. Die Organisationen müssen immer wirksamere

20 Adèle Woods, Les ONG européennes actives dans le domaine du développement. Etat des lieux, op. cit.

${ }^{21}$ Ministère des affaires étrangères, Organisations de solidarité internationale et pouvoirs publics en Europe. Etude comparative sur les dispositifs d'appui et de concertation. Note de synthèse, Paris, Direction générale de la coopération internationale et du développement, Juni 2001, 40 S. 
Marketingmethoden einführen, um das Geld der potenziellen Spender ,anzulocken“. Es wurde bereits erwähnt, dass die „traditionellen“ Hilfsorganisationen der Konkurrenz neuer NRO ausgesetzt sind, die in der Schweiz entstanden sind. Neue Akteure sind auf dem Markt aufgetreten, insbesondere grosse internationale nichtstaatliche Organisationen angelsächsischer Herkunft, die ein aggressiveres Marketing anwenden.

Mehrere NRO, die ihren Sitz im Ausland haben, haben bereits in den 80er Jahren eine Sektion in der Schweiz eingerichtet, namentlich um Spenden anzuziehen, welche anschliessend an das „Mutterhaus“ in den Vereinigten Staaten oder anderswohin überwiesen werden, und in vom Ausland aus verwaltete Projekte fliessen. Die Stiftung ZEWO hatte vor einigen Jahren auf die mit der Gründung von Zweigstellen ausländischer NRO verbundenen Probleme hingewiesen: Abhängigkeit vom Ausland, Lücken in der Transparenz betreffend die Verwendung der in der Schweiz zusammengetragenen Mittel (die Sekretariate in der Schweiz können nicht immer angeben, wofür die Gelder verwendet werden), besonders hohe Kosten für Werbung und für die Finanzierung von Sammelkampagnen $^{22}$. Die Sammelkampagnen in der Schweiz einiger im Ausland niedergelassener Organisationen (z. B. World Vision, Korean Relief und Inter Aid) wurden in der Presse regelmässig herausgestellt ${ }^{23}$. Vertreter traditioneller NRO beklagen sich immer wieder über zunehmende Schwierigkeiten, die Geldgeber zu halten und neue Spender anzuziehen, wie auch über die steigenden Kosten der Sammelkampagnen (oder des relativ geringen „Nutzens“ gewisser Kampagnen). Über Marketingstudien hinaus, die von einigen NRO bei Meinungsforschungsinstituten in Auftrag gegeben werden, gibt es noch wenig akademische Forschungsarbeiten zu diesem Thema ${ }^{24}$.

Das Beispiel von Dr. Richner und seiner Stiftung Kinderspital Kantha Bopha für Kambodscha (mit 18 Millionen Franken an privaten Mitteln im Jahr 2002) ist interessant zu erwähnen. Dr. Richner hat das Ziel, mit seiner stark mediatisierten Sammelkampagne im Sommer 2004 (mit dem Slogan: Eine Million Spenderinnen und Spender, die je 20 Franken geben - Aktion „Zwänzgernötli“) 20 Millionen Franken aufzubringen. Gemäss den Ende Oktober 2004 in der Presse ${ }^{25}$ angekündigten Ergebnissen wurden dank dieser Kampagne 15 Millionen Franken gesammelt, was rund 5 Prozent des gesamten Spendenmarkts für Projekte im Süden und Osten aller schweizerischen NRO für ein Jahr entspricht.

Die Kosten zur Finanzierung der Sammelkampagnen bleiben ein Tabuthema in der Schweiz, und die in den Tätigkeitsberichten der NRO bereitgestellten Angaben sind sehr lückenhaft. Es ist interessant, die Kosten der Sammelkampagnen

22 Quelle: Äusserungen von Robert Zaugg, von der Stiftung ZEWO, die in der Publikation Der Schweizerische Beobachter vom 14. Mai 1993 (Dossier „Entwicklungshilfe: Vermarktung des Elends“) wiedergegeben wurden.

23 Siehe zum Beispiel Leben + Glauben, 2. September 1988; Der Schweizerischer Beobachter, 14. Mai 1993, op. cit.

24 Siehe beispielsweise Myriam Donsimoni, Du don à l'aide. Le marché de l'altruisme, L'Harmattan, Paris, 1995, 228 S.

Florence Passy, L'action altruiste. Contraintes et opportunités de l'engagement différencié, Doktorarbeit an der Universität Genf, Fakultät der Wirtschafts- und Sozialwissenschaften, Universität Genf, 1996.

Maud Gerber, Le don aux ONG et les motivations des donateurs, Diplomarbeit am iuéd, iuéd, Genf, 2000, $44 \mathrm{~S}$.

25 Neue Zürcher Zeitung, 23.-24. Oktober 2004. 
den Einnahmen einer Organisation in Form von Spenden gegenüberzustellen. Das Schweizerische Rote Kreuz gab beispielsweise für 2002 Ausgaben von 2,5 Millionen Franken, bei Spendeneinnahmen von insgesamt 18 Millionen Franken bekannt (Fundraisingkosten von 2,3 Millionen Franken für die Stiftung Kinderdorf Pestalozzi, bei Spenden in Höhe von 6,5 Millionen, und Fundraisingkosten von 2 Millionen Franken für Helvetas, bei Spenden von 8,2 Millionen) ${ }^{26}$. Einige Organisationen verfolgen eine Politik vollkommener Transparenz und veröffentlichen detaillierte Angaben über die Kosten der Sammelkampagnen, zum Beispiel Swissaid, die Stiftung Terre des Hommes Lausanne, Handicap International, ADRA und Mission Bethlehem Immensee (die Rechnungslegung einiger dieser Organisationen ist im Anhang III aufgeführt). Die meisten Organisationen lassen diese Kosten aber in anderen Rubriken der Verwaltungskosten verschwinden.

\section{Die Medien und die Finanzierung der humanitären Hilfe durch die NRO}

Seit einigen Jahren unterscheiden die zur Verfügung stehenden Statistiken über die Hilfe der NRO nicht mehr zwischen humanitärer Hilfe und langfristiger Entwicklungszusammenarbeit. Die letzten erhältlichen Daten zeigten, dass die humanitäre Hilfe im Jahr 1997 rund ein Viertel der gesamten Hilfe der NRO ausmachte, was den Anteil der humanitären Hilfe des Bundes übertrifft, welche rund 20 Prozent der gesamten öffentlichen Entwicklungshilfe ausmacht ${ }^{27}$. Es scheint leichter zu sein, Geld anlässlich mediatisierter Katastrophen, als für gewisse langfristige Projekte zu sammeln. Eine schweizerische Zeitschrift für Entwicklungspolitik brachte 1997 sogar den Titel „Unsere tägliche Katastrophe gib uns heute“28.

Die Glückskette macht durch ihre Spendenaufrufe bei Naturkatastrophen und menschlichen Dramen einen sehr grossen Anteil des Spendenmarktes in der Schweiz aus. Im Jahr 2003 sammelte die Glückskette Gelder in Höhe von insgesamt 15,5 Millionen Franken (2002 : 20,9 Millionen, 2001 : 26,8 Millionen). Diese Beträge sind von einem Jahr zum anderen sehr unterschiedlich. Sie lagen in den letzten Jahren zwischen 10 und 20 Millionen Franken, mit zwei aufeinanderfolgenden Rekordaufkommen von 56 und 57 Millionen Franken in den Jahren 1998 und 1999 für Hurrikan Mitch und für den Krieg im Kosovo.

Die grössten Sammelkampagnen der Glückskette in den letzten Jahren waren folgende $^{29}$ (in Klammern das Hauptjahr der Kampagne und der gesammelte Betrag):

- Unwetter in der Schweiz (2000 : 71,7 Millionen Franken);

- Krieg im Kosovo (1999 : 49,3 Millionen Franken);

- Hurrikan Mitch in Mittelamerika (1998 : 34,4 Millionen Franken);

- Erdbeben in Indien (2001 : 14,3 Millionen Franken);

- Konflikt im Südsudan (1998 : 13,9 Millionen Franken);

26 Neue Zürcher Zeitung, 14. Juli 2003.

27 iuéd, Schweizerische Hilfe für Entwicklungsländer und Oststaaten 1996/1997, Genf, iuéd, 1998. Siehe auch Gérard Perroulaz, „Entwicklung der humanitären Hilfe des Bundes und der schweizerischen Hilfswerke (von 1990 bis 1997)“, in Jahrbuch Schweiz-Dritte Welt 1999 (Dossier: Die Schweiz und die humanitäre Aktion), Genf, iuéd, 1999, S. 9-23.

28 „Unsere tägliche Katastrophe gib uns heute...“ in Mosquito Nr. 4, 1997, Dossier „Spendenmarkt Schweiz".

29 Quelle: Jahresberichte der Glückskette 1996 bis 2003. 
- Überschwemmungen in Mosambik (2000 : 11,3 Millionen Franken);

- Überschwemmungen in Europa (2002 : 7,7 Millionen Franken);

- Kampagne für Kinder als Kriegsopfer (2003 : 7,1 Millionen Franken);

- Erdbeben in Mittelamerika (2001 : 5,5 Millionen Franken);

- Dritte-Welt-Partnerschaften (1997 : 5,2 Millionen Franken);

- Konflikt in Ex-Jugoslawien (1996 : 4,4 Millionen Franken);

- Unwetter in der Schweiz (2002: 4,4 Millionen Franken);

- Überschwemmungen in Osteuropa (1997 : 4,1 Millionen Franken).

Die für Sammelkampagnen „ergiebigsten“ Themen sind deutlich die Überschwemmungen in der Schweiz und im übrigen Europa sowie Naturkatastrophen und Kriegskonflikte. Hungersnot ist eine Geissel, die bei der Kapazität, Spendenmittel aufzubringen, weit hinten anfällt. Die Glückskette überweist die Beträge der Sammlungen an die wichtigsten schweizerischen Organisationen für humanitäre Hilfe und bildet somit eine wichtige Finanzquelle für diese Organisationen. So konnte die Glückskette im Jahr 2003 folgende Summen auszahlen: 9,9 Millionen Franken an Caritas Schweiz, 7,2 Millionen Franken an das Schweizerische Rote Kreuz, 4,9 Millionen Franken an das Hilfswerk der Evangelischen Kirchen der Schweiz, 2,7 Millionen Franken an das Schweizerische Arbeiterhilfswerk, 1,6 Millionen Franken an Terre des Hommes Lausanne und 1,1 Millionen Franken an Medair ${ }^{30}$.

Eine Mitte der 90er Jahre durchgeführte Studie hatte die Prioritäten der schweizerischen Spenderinnen und Spender identifiziert ${ }^{31}$. Die repräsentativ befragten Personen erklärten sich einverstanden, regelmässig Geld für folgende Bereiche (in absteigender Reihenfolge) zu spenden: Kinderhilfe, Behinderte in der Schweiz, Naturschutz, Katastrophenhilfe in der Schweiz, Hilfe für Betagte, Entwicklungshilfe, Kampf gegen Krankheiten, Hilfe für Bergbewohner, Kampf gegen Hunger, kirchliche Missionen, Katastrophenhilfe im Ausland. Am Schluss der Liste standen Hilfe für Drogenabhängige, Hilfe für die Flüchtlinge in der Schweiz und Hilfe für Sozialwerke zugunsten von Frauen.

Die Tatsache, dass die Statistiken über die Hilfe der schweizerischen NRO seit einigen Jahren nicht mehr den Unterschied zwischen kurzfristiger humanitärer Hilfe und Entwicklungszusammenarbeit machen, erklärt sich aus der verschwommenen Abgrenzung zwischen den beiden Arten von Hilfe. In der Tat besteht keine Definition, die zwischen den beiden Einsatzbereichen klar unterscheidet $^{32}$, und ein Teil der als humanitäre Organisationen eingestuften NRO führen auch langfristige Aktionen durch. Wenn die Entsendung von Decken, medizinischer Ausrüstung und Nahrungsmittelhilfe nach einer Katastrophe klar als humanitäre Hilfe eingestuft werden kann, so ist die Grenze zwischen Nothilfe, Wiederaufbau und langfristiger Hilfe verschwommen, wenn zum Beispiel eine nichtstaatliche Organisation eine Schule wieder aufbaut und ausstattet. Eine Reihe von Einsatzbereichen liegt zwischen Nothilfe und langfristiger

30 Weitere Beiträge der Glückskette ergingen an folgende schweizerische Organisationen: Ärzte ohne Grenzen Schweiz, Swissaid, Christlicher Friedensdienst, Enfants du Monde, Handicap International, Terre des Hommes Schweiz, Stiftung Kinderdorf Pestalozzi, Iamaneh Schweiz und Helvetas.

31 „Spendenmarkt Schweiz“, eine Jubiläumsstudie des Verbandes Schweizer Marketing- und Sozialforscher, Basel, 1994.

32 Siehe Gérard Perroulaz, „Entwicklung der humanitären Hilfe des Bundes und der schweizerischen Hilfswerke (von 1990 bis 1997)“, in Jahrbuch Schweiz-Dritte Welt 1999, op. cit. 
Entwicklungszusammenarbeit: Konfliktverhütung, friedenserhaltende Operationen, Hilfe für Strassenkinder, Verteilung von Milch und Nahrung im Rahmen der Unterstützung von Schulen, Minenräumaktionen, Wiedereingliederung von demobilisierten Soldaten oder von Rebellen ins Zivilleben, Unterstützung des Demokratisierungsprozesses, Organisation von Wahlen und Stärkung der Justizsysteme nach einem Bürgerkrieg, Förderung der Menschenrechte in Konfliktgebieten und Unterstützung von Frauen und Kindern als Konfliktopfer.

\section{Relativ geringe Verankerung der Entwicklungszusammenarbeit in der Privatwirtschaft}

Viele grosse schweizerische NRO haben eine starke Verankerung in der Schweizer Bevölkerung, was sich in der Anzahl ihrer Mitglieder oder ihrer regelmässigen Spender ausdrückt. Diese Verankerung stärkt die Legitimität der betreffenden Organisationen. Hingegen scheinen die Beziehungen der NRO zu den privaten Unternehmen sehr schwach zu sein. Ausser ihren Beiträgen zu mediatisierten Sammelaktionen, wie denen der Glückskette, sind die Unternehmen in der Entwicklungszusammenarbeit nur als Materiallieferanten oder Konsulenten präsent. Swisscontact ist ein gutes Beispiel dafür. Die Organisation wurde 1959 gegründet und hat sich auf die Unterstützung kleiner und mittlerer Unternehmen in den Entwicklungsländern sowie auf die Berufsausbildung in diesen Ländern spezialisiert. Die Aktivitäten von Swisscontact werden mehrheitlich durch staatliche Subventionen (DEZA, Kantone und Gemeinden) und sehr wenig durch Spenden privater Unternehmen finanziert. Laut dem Jahresbericht von Swisscontact (siehe Anhang III) stammen über 81 Prozent der Finanzierung dieser Organisation aus staatlichen Subventionen. Die privaten Spenden seitens Unternehmen machen kaum 5 Prozent der Einnahmen aus (2003 : 1,7 Millionen Franken), was für eine Organisation, die das Interesse der Privatwirtschaft für die Entwicklungszusammenarbeit herausstellen sollte, recht wenig ist.

\section{Welche Unabhängigkeit der NRO gegenüber der öffentlichen Hand?}

Die starke Abhängigkeit der NRO gegenüber der Finanzierung durch den öffentlichen Sektor wirft die Frage nach der Handlungsfreiheit dieser Organisationen und ihrer Fähigkeit auf, kritische Positionen gegenüber der Aussenpolitik der Schweiz, insbesondere bezüglich der Politik der Entwicklungszusammenarbeit, einzunehmen.

Gewisse konservative Kreise, darunter die Schweizerische Volkspartei (SVP), üben auf die politischen Behörden Druck aus, um zu vermeiden, dass die DEZA Gelder an Organisationen zahlt, welche die Regierungspolitik kritisieren. Vorwürfe in diesem Sinne wurden in der Schweiz bei Debatten über die Abschaffung der Armee oder über die Kürzung der Militärausgaben formuliert. Welche kritische Distanz können die NRO einnehmen, wenn die privaten Konsulenten, mit denen sie in Konkurrenz stehen, nicht die Bestimmung haben, öffentlich Stellung zu diesen Fragen zu beziehen? Besteht nicht die Gefahr, dass diejenigen NRO, die am stärksten von der öffentlichen Hand abhängig sind, zu quasiöffentlichen oder halbstaatlichen Organisationen werden? Die diesbezügliche Debatte ist lebhaft, wobei einige Autoren behaupten, dass die öffentliche Finan- 
zierung die NRO mundtot mache, während andere der Meinung sind, dass die NRO sich ihre Unabhängigkeit bewahren können und dabei gleichzeitg dem Staat ihre Kompetenzen anbieten können ${ }^{33}$.

Eine genaue Analyse der Auswirkungen, die der Umfang der öffentlichen Finanzierung auf die Unabhängigkeit der Stellungnahmen der NRO hat, muss noch erfolgen, jedoch kann man die Annahme machen, dass es auf diese Frage keine einfache und eindeutige Antwort gibt. Auch ist $\mathrm{zu}$ betonen, dass die Abhängigkeit einer nichtstaatlichen Organisation gegenüber den privaten Geldgebern nicht ohne Bedeutung ist. In der Tat können einige Spender in Bezug auf Stellungnahmen zu Themen wie politisches Asyl oder Bankgeheimnis sehr sensibel sein.

Man kann keinen klaren Zusammenhang zwischen einem hohen Anteil an öffentlicher Finanzierung der Nichtregierungsorganisationen in einem gegebenen Land und fehlender Unabhängigkeit der NRO herstellen. Die französischen NRO werden vom eigenen Staat wenig, von der Europäischen Union jedoch reichlich finanziert. Kann man daraus folgern, dass diese Organisationen unabhängiger seien als die NRO in der Schweiz? Die Abhängigkeit der Organisationen, welche Mitglieder der Arbeitsgemeinschaft der Hilfswerke sind, gegenüber der öffentlichen Finanzierung hindert die Arbeitsgemeinschaft nicht daran, die ungehinderte Liberalisierung der internationalen Wirtschaftsbeziehungen zu kritisieren und dabei gleichzeitig die Politik der DEZA in anderen Bereichen zu unterstützen.

\section{Betriebsausgaben der NRO in der Schweiz}

Die oben im Abschnitt über die öffentliche Finanzierung der NRO analysierten Daten betreffen nur die Ausgaben in den Entwicklungs- und Transitionsländern. Sie umfassen weder die Verwaltungskosten der Sekretariate der NRO in der Schweiz, noch die Kosten für die Informationsbulletins in der Schweiz und die Sammelkampagnen (Fundraising).

Die Geber wünschen in den meisten Fällen, dass die Gesamtheit ihrer Spenden für Projekte vor Ort verwendet werden. Dieser Wunsch, so verständlich er ist, steht im Konflikt mit einem anderen Anliegen des Spenders, nämlich dass seine Beiträge so wirksam wie möglich eingesetzt werden ${ }^{34}$. Der Professionalismus des Personals, um eine effiziente Projektbegleitung, eine strikte Rechnungsführung, die Abfassung von Berichten und die Evaluierung der Projekte zu gewährleisten, verlangt von den NRO einen leistungsfähigen technischen, Finanz- und Verwaltungsapparat und somit die Mobilisierung entsprechender Ressourcen.

Die von den NRO angegebenen Verwaltungskostenanteile müssen mit Vorsicht interpretiert werden, denn es ist keineswegs nachgewiesen, dass eine Organisa-

33 Es besteht eine umfangreiche Literatur zu diesem Thema, jedoch wird insbesondere auf folgende Studien verwiesen: Ian Smillie, Henny Helmich, (Hrsg.), Stakeholders: Government-NGO Partnerships for International Development, London, Earthscan Publications, OECD, 1999, 318 S.; J.-P. Deler, Y.-A. Fauré, A. Piveteau, P.-J. Roca, ONG et développement. Société, économie, politique, Paris, Ed. Karthala, Collection Hommes et sociétés, 1998, 684 S.; Philippe Ryfman, Les ONG, La Découverte, Collection Repères, Paris, 2004, 124 S.

34 Siehe hierzu die Pressemitteilung der Stiftung ZEWO vom 5. Juli 2004, „Verwaltungsaufwand gemeinnütziger Organisationen. Notwendigkeit, seriöse Projekte durchzuführen“. 
tion, die einen geringeren Verwaltungskostenanteil aufführt als eine andere, effizienter ist. Die Angaben der Jahresberichte einiger NRO zeigen, dass der Anteil an Verwaltungs- und Marketingkosten von einer Organisation zur anderen sehr verschieden ist (siehe Anhang III, der Beispiele bezüglich der Aufteilung der Ausgaben und Einnahmen einer Reihe von NRO aufführt). Auch ist die Präsentation der Kostenaufteilung zwischen verschiedenen Rubriken je nach den Organisationen unterschiedlich. Ein Teil der Betriebskosten kann in die Überwachung der Projekte vor Ort eingeschlossen werden, und die Fundraisingkosten werden oft in den allgemeinen Verwaltungskosten (mit den Sekretariatskosten) verbucht oder mit der Information in der Schweiz zusammengelegt. Somit kann es sich bei einer Organisation, die einen relativ grösseren Verwaltungs- und Fundraisingaufwand hat, um eine Organisation handeln, die diese Ausgaben aus Transparenzgründen nicht in anderen Buchungsposten unterbringt. Dank den Empfehlungen in der Rechnungslegung der Swiss GAAP RPC 21 werden in einigen Jahren standardisiertere Daten zur Verfügung stehen ${ }^{35}$.

In der Debatte über den Anteil der Ressourcen der NRO, die in der Schweiz ausgegeben werden, sollte man zudem nicht vergessen, dass die internationale Solidarität auch den Kampf für eine Änderung der in den Wirtschaftsbeziehungen mit den Entwicklungsländern vorherrschenden Bedingungen umfasst. Dies erklärt die Bedeutung der Information in der Schweiz und der Kampagnen für eine bessere Kohärenz der öffentlichen Politiken gegenüber den Entwicklungsländern.

\section{Einige internationale Vergleiche zur Rolle der NRO in der Entwicklungs- zusammenarbeit}

Trotz der Schwierigkeit, die Entwicklungshilfe und die Finanzierung der NRO verschiedener Länder zu vergleichen (nicht harmonisierte Methoden beim Zusammentragen der Daten, Meinungsverschiedenheiten betreffend die Definition einer NRO, Umfrage über die Hilfe der nichtstaatlichen Organisationen, die je nach den Ländern einen mehr oder minder grossen Anteil der gesamten Zahl der NRO abdeckt), erschien es uns nützlich, die Angaben über die Finanzierung der schweizerischen NRO den Daten der anderen Länder des Entwicklungshilfeausschusses der OECD (DAC) gegenüberzustellen.

\section{Statistiken des OECD-Entwicklungshilfeausschusses}

Die DAC-Statistiken unterscheiden zwischen den Leistungen der NRO, welche mit Eigenmitteln finanziert werden ${ }^{36}$, und den Leistungen der NRO, die durch öffentliche Beiträge für die Programme der NRO finanziert werden. Gemäss diesen Statistiken beliefen sich die mit Eigenmitteln der NRO finanzierten Leistungen im Jahr 2002 für die Gesamtheit de DAC-Länder auf 8,8 Milliarden Dollar und die mit öffentlichen Mitteln finanzierten Leistungen auf 1,2 Milliarden Dollar. Die öffentlichen Beiträge an die NRO hatten in den 80er Jahren stark zugenommen, um dann von 1990 bis 1998 bei einer Milliarde Dollar zu stagnie-

35 Empfehlungen, die von den Organisationen mit dem ZEWO-Gütesiegel eingehalten werden müssen.

36 Spenden der privaten freiwilligen Organisationen. 
ren. Die höchsten Dollarbeiträge pro Einwohner wurden 2002 von Norwegen (rund 100 Dollar pro Einwohner) geleistet. Die Schweiz steht mit einem Durchschnitt von 28 Dollar pro Einwohner an zweiter Stelle (Tabelle 3).

Diese statistischen Daten sind jedoch partiell, da das DAC weder die Angaben über die den NRO erteilten spezifischen Aufträge, noch die Beiträge des Staates für sämtliche humanitären Einsätze der NRO veröffentlicht. Die DAC-Daten sind nicht vollständig und nicht immer unter den Ländern vergleichbar, weil jede Regierungspolitik der Zusammenarbeit mit den NRO von der betreffenden Politik der anderen Länder sehr verschieden ist.

Tabelle 3: Spenden der privaten freiwilligen Organisationen der DAC-Länder in die Entwicklungsländer, 2002 (in Millionen Dollar und in Dollar pro Einwohner)

\begin{tabular}{lrrr}
\hline Land & $\begin{array}{c}\text { Private Spenden } \\
\text { in die Entwicklungsländer }\end{array}$ & $\begin{array}{r}\text { Private Spenden } \\
\text { in die Transitionsländer }\end{array}$ & $\begin{array}{r}\text { in Dollar pro } \\
\text { Einwohner }\end{array}$ \\
\hline Australien & 248 & 248 & 12.7 \\
\hline Österreich & 57 & 8 & 7.1 \\
\hline Belgien & 74 & 10 & 7.2 \\
\hline Kanada & 276 & 2 & 8.8 \\
\hline Dänemark & 17 & 78 & 3.2 \\
\hline Finnland & 10 & 1.9 \\
\hline Deutschland & 823 & & 10.0 \\
\hline Griechenland & 6 & & 0.5 \\
\hline Irland & 86 & 22.2 \\
\hline Italien* & 32 & 0.6 \\
\hline Japan & 157 & 1.2 \\
\hline Luxemburg & 2 & & 4.5 \\
\hline Niederlande & 257 & & 15.9 \\
\hline Neuseeland & 23 & 5.8 \\
\hline Norwegen & 452 & 9 & 99.3 \\
\hline Portugal & 5 & 6 & 0.5 \\
\hline Schweden & 19 & 3146 & 2.1 \\
\hline Schweiz & 202 & $\mathbf{3 5 0 8}$ & 27.6 \\
\hline Grossbritannien & 353 & 6.0 \\
\hline USA & 5720 & 19.8 \\
\hline DAC insgesamt & $\mathbf{8 7 6 5}$ & $\mathbf{1 0 . 2}$ \\
\hline
\end{tabular}

* Spenden für 2001, wenn Angaben für 2002 nicht erhältlich.

Quelle: OECD, DAC-Bericht. Coopération pour le développement, 2002.

Die Spenden der privaten freiwilligen Organisationen der Mitgliedsstaaten des DAC machen im Jahr 2002 0,05 Prozent des Bruttonationaleinkommens der DAC-Länder aus, und diese 8,8 Milliarden kommen zu den 58,3 Milliarden Dollar öffentlicher Entwicklungshilfe hinzu, die 0,23 Prozent des BNE ausmachen (siehe Tabelle 4).

Aus der DAC-Statistik sind auch die Beiträge des Zentralstaates an die NRO ersichtlich, jedoch betreffen die Angaben nur die Beiträge an Programme, die von den NRO ausgewählt und durchgeführt werden (z.B. ohne Regieprojekte). Diese öffentlichen Beiträge an die NRO beliefen sich 2002 auf 1246 Millionen Dollar.

Die Angaben der umseitigen Tabelle 4 zeigen ferner, dass sich die Beiträge der privaten Organisationen in den letzten Jahren deutlich erhöht haben. Hierzu ist zu vermerken, dass die Hilfe der NRO rascher als die öffentliche Entwicklungshilfe gestiegen ist. Die Leistungen der NRO (an die Entwicklungs- und Transitionsländer) haben sich von 1989 bis 2002 verdreifacht und sind von 4 Milliarden Dollar 
auf über 12 Milliarden Dollar im Jahr 2002 angestiegen, wogegen die öffentliche Entwicklungshilfe im gleichen Zeitraum lediglich um 27 Prozent zunahm.

Tabelle 4: Entwicklung der privaten Spenden der freiwilligen Organisationen der DAC-Länder, 1981-2002 (in Millionen Dollar)

\begin{tabular}{|c|c|c|c|c|c|}
\hline & $\begin{array}{l}\text { Private Spenden an } \\
\text { die Entwicklungsl. } \\
\text { DAC-Liste 1. Teil* }\end{array}$ & $\begin{array}{l}\text { Private Spenden an } \\
\text { die Transitionsländer } \\
\text { DAC-Liste 2. Teil* }\end{array}$ & $\begin{array}{c}\text { Private Spenden } \\
\text { insgesamt }\end{array}$ & $\begin{array}{l}\text { Beiträge der Staaten } \\
\text { an die NRO (in der } \\
\text { öff. EH enthalten) }\end{array}$ & $\begin{array}{r}\text { Öffentl. Ent- } \\
\text { wicklugshilfe } \\
\text { zum Vergleich }\end{array}$ \\
\hline 1981-1983 & 2213 & & 2213 & n.e. & 26134 \\
\hline 1983-1984 & 2458 & & 2458 & 32 & 27450 \\
\hline 1984-1985 & 2741 & & 2741 & 203 & 28443 \\
\hline 1985-1986 & 3109 & & 3109 & 250 & \\
\hline 1986-1987 & 3674 & & 3674 & 600 & 38221 \\
\hline 1987-1988 & 4124 & & 4124 & 647 & 43834 \\
\hline 1990 & 5077 & & 5077 & 1001 & 52955 \\
\hline 1991 & 5403 & 247 & 5650 & 972 & 56678 \\
\hline 1992 & 6005 & 204 & 6209 & 885 & 60850 \\
\hline 1993 & 5692 & 425 & 6117 & 874 & 56498 \\
\hline 1994 & 6046 & 411 & 6457 & 972 & 59152 \\
\hline 1995 & 5973 & 398 & 6371 & 1053 & 58926 \\
\hline 1996 & 5568 & 379 & 5947 & 1010 & 55438 \\
\hline 1997 & 5191 & $1161^{* *}$ & 6352 & 998 & 48324 \\
\hline 1998 & 5609 & 1554 & 7163 & 1037 & 51888 \\
\hline 1999 & 6715 & 2232 & 8947 & 1151 & 53233 \\
\hline 2000 & 6934 & 2524 & 9458 & 1200 & 53749 \\
\hline 2001 & 7289 & 3151 & 10440 & 1137 & 52335 \\
\hline 2002 & 8765 & 3508 & 12273 & 1246 & 58274 \\
\hline $\begin{array}{l}\text { in \% des BNE } \\
\text { (2002) }\end{array}$ & 0,035 & & 0,049 & & 0,235 \\
\hline
\end{tabular}

* Die DAC-Liste der Hilfeempfängerländer wird jedes Jahr vom Entwicklungshilfeausschuss der OECD (DAC) festgelegt.

** Seit 1997 ist ein grosser Teil der Erhöhung der Spenden an die Transitionsländer auf die Verlegung gewisser Länder (z.B. Israel) vom 1. Teil der DAC-Liste (Entwicklungsländer) in den 2. Teil (Transitionsländer) zurückzuführen.

n.e. $=$ nicht erhältlich .

Quelle: OECD, DAC-Bericht, Coopération pour le développement, verschiedene Jahrgänge.

Die Höhe der staatlichen Unterstützung der NRO in der öffentlichen Entwicklungshilfe ist von einem Land zum anderen sehr unterschiedlich. Der Anteil der öffentlichen Finanzierung der NRO an der gesamten nationalen öffentlichen Entwicklungshilfe lag 1998 zwischen 12 Prozent in Luxemburg und 0,6 Prozent in Frankreich (Schweiz 4,8\%) ${ }^{37}$.

\section{Frankreich - Finanzierung der Organisationen für internationale Solidarität}

In Frankreich werden von der Kommission Entwicklungszusammenarbeit (Commission Coopération-Développement) alle zwei Jahre Erhebungen durchgeführt, um die Bedeutung der Nichtregierungsorganisationen in der Entwicklungszusammenarbeit aufzuzeigen. Die letzte verfügbare Umfrage bezieht sich auf das Jahr 200138. Im Rahmen dieser Erhebung wurden Fragebögen an 2000 französische Organisationen für internationale Solidarität versandt. Die Ergeb-

37 Quelle: Ministère des affaires étrangères, Organisations de solidarité internationale et pouvoirs publics en Europe, Etude comparative sur les dispositifs d'appui et de concertation. Note de synthèse, op. cit.

38 Commission Coopération-Développement. Argent et organisations de solidarité internationale, Paris, Dezember 2003, 32 S. 
nisse beruhen auf den Angaben von 157 Organisationen, welche praktisch alle Vereinigungen von nationaler Tragweite und Bekanntheit umfassen. Die grössten französischen NRO sind hauptsächlich in der Nothilfe tätig: Médecins sans frontières (1999: Budget von 70 Millionen Euro), Médecins du Monde (51 Millionen), das französische Komitee für UNICEF (48,5 Millionen), Handicap International (44 Millionen), Action contre la faim (40 Millionen), Secours catholique (34 Millionen) und das Comité contre la faim et pour le développement (31 Millionen) $)^{39}$.

\section{$\square$ Finanzmittel der französischen NRO}

Tabelle 5: Finanzmittel und Ausgaben der französischen NRO, 2001 (in Millionen Euro und in \%)

\begin{tabular}{lrr}
\hline Finanzmittel der Organisationen & in Millionen Euro & in \% des Gesamtbetrags \\
\hline Private Mittel & & \\
\hline Spendenerträge der Öffentlichkeit & 314,3 & 44,1 \\
\hline Unternehmer-Mäzenatentum & 9,2 & 1,3 \\
\hline Patenschaften & 5,0 & 0,7 \\
\hline Sonstige private Beiträge (Stiftungen, NRO, usw.) & 27,4 & 3,8 \\
\hline Beiträge und Abonnements & 5,4 & 0,8 \\
\hline Verkaufs- und Betriebserträge & 11,3 & 1,6 \\
\hline Finanzielle und sonstige Erträge & 67,3 & 9,4 \\
\hline Private Mittel Zwischensumme & 440,0 & 61,7 \\
\hline Öffentliche Mittel & & 5,7 \\
\hline Aussenministerium/Ministerium für Entwicklungszusammenarbeit & 40,6 & 2,7 \\
\hline Andere nationale staatliche Subventionen & 19,6 & 17,3 \\
\hline Europäische Union & 123,6 & 3,8 \\
\hline Vereinte Nationen & 27,2 & 5,0 \\
\hline Sonstige internationale Beihilfen & 35,3 & 1,0 \\
\hline Regionale Beihilfen (dezentralisierte Zusammenarbeit) & 6,9 & 2,7 \\
\hline Vom nationalen oder internationalen öffentlichen Sektor & & 38,3 \\
finanzierte Aufträge & 19,5 & \\
\hline Öffentliche Mittel Zwischensumme & 272,7 & $\mathbf{7 1 2 , 7}$ \\
\hline Gesamtbetrag der Mittel & & \\
\hline
\end{tabular}

\begin{tabular}{lrr}
\hline \multicolumn{1}{l}{ Ausgaben der Organisationen } & in Millionen Euro & in \% des Gesamtbetrags \\
\hline Tätigkeit vor Ort & & \\
\hline \multicolumn{1}{l}{ Von der französischen NRO gezahlte lokale Gehälter } & 44,1 & 6,2 \\
\hline Gehälter der Mitarbeiter im Ausland & 52,4 & 7,4 \\
\hline Kosten für Einsatzbeauftragte und Experten & 15,4 & 2,2 \\
\hline Projektkosten (Material, usw.) & 240,5 & 33,7 \\
\hline$\quad$ Unterstützung lokaler Organisationen & 54,6 & 7,7 \\
\hline Zahlungen an andere NRO oder internationale Organisationen & 9,7 & 1,4 \\
\hline$\quad$ Sonstige Ausgaben vor Ort & 83,3 & 11,7 \\
\hline Tätigkeit vor Ort Zwischensumme & 500,0 & 70,2 \\
\hline Tätigkeit in Frankreich & 28,1 & 3,9 \\
\hline$\quad$ Information, Sensibilisierung, Entwicklungsbildung & 7,9 & 1,1 \\
\hline$\quad$ Personalausbildung, Anstellung, Praktika, Stipendien, usw. & 113,6 & 15,9 \\
\hline Betriebskosten & 61,6 & $\mathbf{8 , 6}$ \\
\hline Sammelspesen & $\mathbf{1 , 4}$ & $\mathbf{0 , 2}$ \\
\hline Verschiedene Ausgaben in Frankreich & $\mathbf{7 1 2 , 6}$ & \\
\hline Ausgaben Gesamtbetrag & &
\end{tabular}

Quelle: Argent et organisations de solidarité internationale 2000-2001, Paris, Commission Coopération-Développement, 2003, 32 S.

39 Quelle: Ministère des affaires étrangères, Organisations de solidarité internationale et pouvoirs publics en Europe. Etude comparative sur les dispositifs d'appui et de concertation. Note de synthèse, op. cit. 
Gemäss der im Jahr 2003 veröffentlichten Erhebung der Kommission Entwicklungszusammenarbeit belief sich der Gesamthaushalt der im Bereich der internationalen Solidarität tätigen französischen NRO im Jahr 2001 auf 712,7 Millionen Euro. Die privaten Mittel beliefen sich auf 440 Millionen Euro (d.h. $62 \%$ der gesamten Ressourcen) und die öffentlichen Mittel machten 38 Prozent der gesamten Ressourcen aus (siehe Tabelle 5).

\section{Grafik 3: Aufteilung der Finanzmittel der französischen NRO}

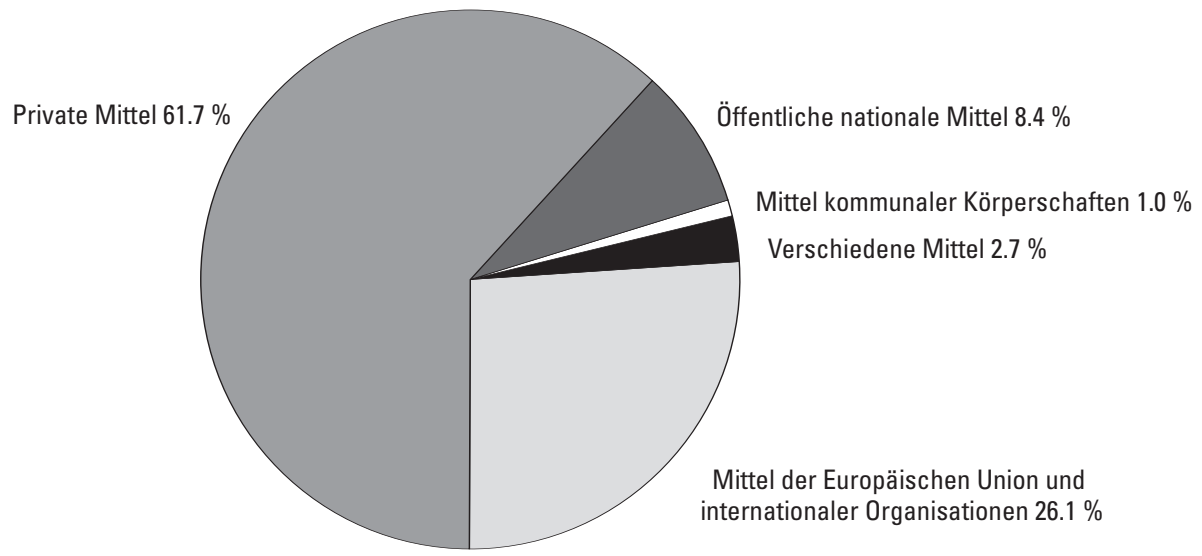

Quelle: Argent et organisations de solidarité internationale 2000-2001, Paris, Commission Coopération-Développement, 2003, 32 S.

Die öffentlichen Mittel der französischen Organisationen, die 2001 insgesamt 273 Millionen Euro ausmachten, stammen vor allem aus internationalen Mitteln (68\% der öffentlichen Ressourcen), aus Mitteln des Zentralstaates (22\%) und aus Mitteln öffentlicher Gebietskörperschaften (2,5\%), der Rest besteht aus verschiedenen Mitteln (7\%). Bei den internationalen Mitteln machten die Beiträge der Europäischen Union mit 124 Millionen Euro im Jahr 2001 (45\% aller öffentlichen Ressourcen französischer NRO) den grössten Anteil aus.

\section{$\square$ Ausgaben der französischen NRO}

Die in Frankreich durchgeführten Umfragen zeigen die Zusammensetzung der Ausgaben der NRO genauer auf als in der Schweiz. Die Gesamtaufwendungen der französischen NRO beliefen sich 2001 auf 712 Millionen Euro. Der Anteil der Tätigkeit in Drittländern macht 70 Prozent der gesamten Ausgaben der NRO, das heisst 500 Millionen Euro aus (wogegen es in der Schweiz keine globalen Daten über den Anteil der Ausgaben der schweizerischen NRO gibt). Grafik 4 (S. 73) führt die Zusammensetzung der Ausgaben der französischen NRO auf.

Die verfügbaren Zahlenangaben lassen sich wie folgt kommentieren :

• Die Unterstützung lokaler Organisationen in den Empfängerländern der Hilfe bleibt relativ gering, trotz des vorherrschenden Diskurses, welcher der Unterstützung lokaler Initiativen, der Partizipation der betreffenden Bevölkerungsgruppen, der Partnerschaft mit den Organisationen des Südens und der notwendigen Stärkung der Kapazitäten der Zivilgesellschaft im Süden grossen 
Wert beimisst. Im Jahr 2001 wurden lediglich 55 Millionen Euro, das heisst 7,7 Prozent der Gesamtaufwendungen der französischen NRO, an lokale Organisationen ausgezahlt.

๑ Die Gehaltszahlungen im Zusammenhang mit Projekten im Ausland machen rund 16 Prozent der Ausgaben der NRO aus: 44 Millionen Euro für lokale Gehälter, 52 Millionen für das ins Ausland entsandte Personal (darunter die Freiwilligen) und 15 Millionen für Evaluierungseinsätze und Projektbegleitung.

๑ Die Aufwendungen betreffend die Sensibilisierung für Entwicklungsfragen, die Information in Frankreich und die Entwicklungsbildung machen 5 Prozent der Gesamtausgaben aus (in der Schweiz gibt es keine vergleichbaren Angaben).

- Die Betriebsausgaben der NRO-Sekretariate in Frankreich machen 15,9 Prozent der Gesamtaufwendungen aus (keine vergleichbaren Angaben in der Schweiz).

$\checkmark$ Die mit den Sammelkampagnen verbundenen Kosten sind ein wichtiger Ausgabenposten und beliefen sich 2001 auf 8,6 Prozent der Gesamtaufwendungen der NRO, das heisst 14 Prozent der privaten Einnahmen dieser Organisationen. Es ist relevant, diesen Betrag von 61,6 Millionen Euro den Einnahmen der privaten Spenden gegenüberzustellen (ohne Verkaufserträge, ordentliche Verbandsmitglieder und Unternehmenssponsoring). Somit betragen die Sammelspesen 20 Prozent der Erträge der Sammelkampagnen.

\section{Grafik 4: Aufteilung der Ausgaben der französischen NRO (2001)}

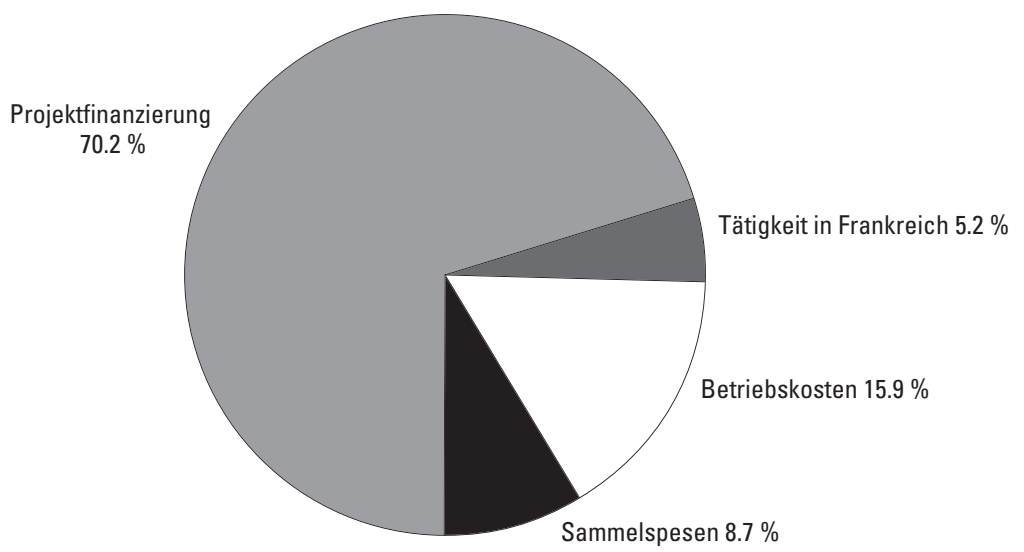

Quelle: Berechnung anhand der Angaben der Publikation der Commission Coopération-Développement: Argent et organisations de solidarité internationale 2000-2001, Paris, 2003, 32 S. 


\section{Deutschland}

In Deutschland gibt es rund 2000 entwicklungspolitische Nichtregierungsorganisationen ${ }^{40}$, von denen neun zu den 25 grössten nichtstaatlichen Organisationen in Europa gehören. Die deutschen NRO lassen sich in drei grosse Gruppen einteilen: mit den Kirchen verbundene NRO, politische Stiftungen, andere nichtkirchliche NRO.

Das Bestehen politischer Stiftungen ist eine Besonderheit in Deutschland. Jede der grossen Parteien hat eine Stiftung für Entwicklungszusammenarbeit, deren Aktivitäten durch öffentliche Mittel finanziert werden. Die wichtigsten Stiftungen sind die Konrad-Adenauer-Stiftung (Christlich Demokratische Union, CDU), die Friedrich-Ebert-Stiftung (Sozialdemokratische Partei, SPD), die Friedrich-Naumann-Stiftung (Freie Demokratische Partei, FDP), die Hanns-SeidelStiftung (Christlich Soziale Union, CSU), die Heinrich-Böll-Stiftung (Grüne) und die Rosa-Luxemburg-Stiftung (Partei des Demokratischen Sozialismus, PDS). Die Stiftungen sollen insbesondere die Festigung demokratischer Strukturen sowie die Stärkung von Gewerkschaften und Parteien ermöglichen: Förderung der Beteiligung der Bevölkerung am politischen Entscheidungsprozess, Ausbildung, Förderung unabhängiger Medien, Unterstützung von Basisorganisationen, Stärkung der Forschungskapazitäten. Die Stiftungen werden vor allem vom Bundesministerium für wirtschaftliche Zusammenarbeit und Entwicklung (BMZ) und vom Bundesinnenministerium finanziert, wobei die Beträge gemäss dem Stimmenanteil bei den Wahlen verteilt werden (die Stiftungen treten nicht direkt durch Sammelkampagnen auf dem Spendenmarkt auf).

Die Leistungen der deutschen NRO, welche durch deren Eigenmittel zugunsten der Entwicklungsländer finanziert werden, beliefen sich im Jahr 2002 auf 874 Millionen Euro ${ }^{41}$. Diese Leistungen hatten sich von 1990 bis 1995 regelmässig erhöht, indem sie von 625 Millionen auf 815 Millionen Euro anstiegen. Man kann einen Rückgang der Leistungen um 6 Prozent von 1999 (wo sie den Rekordbetrag von 931 Millionen Euro erreichten) bis 2002 feststellen. Tabelle 6 (S. 75) gibt eine Übersicht über die privaten Spenden der wichtigsten NRO.

Wie in der Schweiz spielen die den Kirchen nahe stehenden Organisationen Deutschlands in der Entwicklungshilfe eine sehr wichtige Rolle. Sie brachten im Jahr 200249 Prozent der privaten Mittel (bei gleicher Aufteilung zwischen evangelischen und katholischen Organisationen) auf. Die kirchlichen NRO sammeln private Spenden, erhalten öffentliche Beihilfen und Landeskirchensteuern.

40 Gemäss Bundesministerium für wirtschaftliche Zusammenarbeit und Entwicklung, Medienhandbuch Entwicklungspolitik 2004/2005, Berlin, Juni 2004, 520 S. Das Handbuch kann im Internet unter der Adresse : <www.bmz.de> heruntergeladen werden. Es enthält ein Kapitel über die Entwicklungshilfe der NRO und die öffentliche Finanzierung dieser Organisationen. Für eine Übersicht über die Hilfe der NRO in Deutschland und die Beziehungen zwischen NRO und Staat siehe auch Judith Randel und Tony German in: Smillie, Ian; Helmich, Henny, (Hrsg), Stakeholders, Government-NGO Partnerships for International Development, London, Earthscan Publications, op. cit, S. 114-122.

41 Anhand der Informationen von 351 im Bereich der Hilfe für Entwicklungsländer tätigen Organisationen und 134 im Bereich der Hilfe für Transitionsländer tätigen Organisationen. 
Tabelle 6: Private Hilfe (Eigenmittel und private Spenden) der NRO in Deutschland, 2002 (in Millionen Euro)

\begin{tabular}{|c|c|c|}
\hline Nichtregierungsorganisationen & $\begin{array}{c}\text { Spenden für } \\
\text { Transitionsländer }\end{array}$ & $\begin{array}{l}\text { Spenden für Ent- } \\
\text { wicklungsländer }\end{array}$ \\
\hline Evangelische Organisationen insgesamt & 226,4 & 9,1 \\
\hline Diakonisches Werk der EKD & 66,6 & 4,3 \\
\hline Evangelisches Missionswerk in Deutschland & 38,1 & 0,0 \\
\hline Kindernothilfe & 36,5 & 0,9 \\
\hline Evangelischer Entwicklungsdienst & 26,0 & 0,4 \\
\hline Christoffel-Blindenmission & 20,1 & 0,5 \\
\hline Evangelisch-Lutherische Kirche in Bayern & 12,7 & 1,5 \\
\hline Andere evangelische Organisationen & 26,4 & 1,5 \\
\hline Katholische Organisationen insgesamt & 201,1 & 33,5 \\
\hline Misereor & 43,2 & 0,0 \\
\hline Deutscher Caritasverband & 26,6 & 3,8 \\
\hline Adveniat & 24,0 & 0,0 \\
\hline Missio & 19,2 & 0,4 \\
\hline Missionsprokur der Deutschen Jesuiten & 11,1 & 0,4 \\
\hline Verband der Diözesen Deutschlands & 5,4 & 12,7 \\
\hline Renovabis & 0,0 & 9,6 \\
\hline Andere katholische Organisationen & 71,6 & 6,7 \\
\hline Nichtkirchliche Organisationen insgesamt & 446,2 & 40,7 \\
\hline Hermann-Gmeiner-Fonds Deutschland & 98,3 & 7,2 \\
\hline Deutsches Komitee für UNICEF & 59,1 & \\
\hline Plan International Deutschland & 38,8 & \\
\hline Deutsches Rotes Kreuz & 34,0 & 2,5 \\
\hline Deutsche Welthungerhilfe & 22,9 & \\
\hline SOS Kinderdorf & 18,3 & 1,2 \\
\hline World Vision & 17,8 & \\
\hline Deutsche Lepra- und Tuberkulosehilfe & 14,5 & \\
\hline Ärzte ohne Grenzen & 12,8 & \\
\hline Förderkreis für die Schwestern Maria & 11,6 & 3,1 \\
\hline Andere nichtkirchliche NRO & 118,1 & 26,6 \\
\hline Private Spenden der NRO insgesamt & 873,7 & 83,3 \\
\hline
\end{tabular}

Quelle: Bundesministerium für wirtschaftliche Zusammenarbeit und Entwicklung, Tabelle im Internet: <www.bmz.de>, 2004/2005, stat-06c.xls und stat-06d.xls, 10.11.2003.

$\mathrm{Zu}$ dieser privaten Finanzierung der NRO in Höhe von 874 Millionen Euro kommen die NRO-Beiträge des Bundesministeriums für wirtschaftliche Zusammenarbeit und Entwicklung (BMZ) hinzu. Einer Tendenz folgend, die sich auch in der Schweiz abzuzeichnen scheint, haben die privaten Mittel der deutschen NRO von 1990 bis 2002 rascher zugenommen als ihre öffentlichen Mittel (Anstieg der privaten Mittel um $40 \%$ und der staatlichen Beihilfen des BMZ an die NRO um $20 \%)^{42} .1997$ machte die öffentliche Finanzierung 40 Prozent der gesamten Finanzierung der deutschen nichtstaatlichen Organisationen aus ${ }^{43}$.

1988 wurde die Beratungsstelle bengo ${ }^{44}$ eingerichtet, um jene NRO, die weder kirchliche Organisationen noch politische Stiftungen sind, zu beraten und ihnen den Zugang zu öffentlichen Mitteln zu erleichtern.

42 BMZ, Medienhandbuch 2004/2005, Tabelle 17, S. 398.

43 Judith Randel und Tony German, opus cit., S. 144

44 bengo, Beratungsstelle für private Träger in der Entwicklungszusammenarbeit, <www.paritaet.org/ bengo>. 
Ein grosser nationaler Verband (Venro) ${ }^{45}$ vereinigt seit 1995 Hunderte von NRO (ohne politische Stiftungen). Hatten die Prüfungen des OECD-Entwicklungshilfeausschusses DAC bis 1995 noch auf das Fehlen einer Dialogstruktur zwischen der Bundesregierung und den kleinen NRO hingewiesen, so konnte die Situation durch diesen Verband wesentlich verbessert werden.

\section{Schlussbemerkungen}

Der vorliegende Artikel hat die Situation der schweizerischen NRO auf dem Spendenmarkt dargestellt und die Dynamik aufgezeigt, die diese Organisationen antreibt, um die privaten finanziellen Mittel unter sich aufzuteilen. Betreffend die öffentlichen Mittel, über welche die schweizerischen Nichtregierungsorganisationen verfügen, hat der Artikel die polemische Frage der Unabhängigkeit dieser NRO gegenüber den öffentlichen Geldgebern herausgestellt und auf einen noch zu untersuchenden Forschungsbereich hingewiesen.

Es ist festzustellen, dass die privaten Mittel in letzter Zeit stärker angestiegen sind als die öffentlichen Mittel (wie Tabelle 1 für die Schweiz und Tabelle 4 für sämtliche DAC-Länder zeigen). Es scheint, dass der Bund zwar die bedeutende Rolle der NRO in der Aussenpolitik der Schweiz anerkennt, aber seine Beiträge diversifiziert hat und die für die NRO bestimmten Beiträge als Ergänzung zu den privaten Mittel betrachtet hat. Diese Erscheinung ist auch in anderen DAC-Ländern zu beobachten, besonders in Deutschland, wie wir gerade gesehen haben.

Die Analyse der Finanzierungsquellen der Schweizer NRO, die dieser Artikel zusammenfasst, erweist sich als ein sehr interessantes Instrument, um die Komplexität der Arbeit der schweizerischen nichtstaatlichen Organisationen im Bereich der Hilfe an die Entwicklungs- und Transitionsländer zu verstehen. Denn die finanziellen Mittel, über welche die NRO verfügen, ob privater oder öffentlicher Herkunft, sind Bedingungen unterworfen, die nicht immer untereinander vereinbar sind, und dadurch die Leitungsorgane dieser Organisationen zwingen, bei ihrer Arbeit ständig einen Balanceakt zu vollführen.

\section{QUELLEN}

Commissariat général du Plan, L'Etat et les ONG: vers un partenariat efficace. Paris, La Documentation française, 2002, 210 S. Bericht der Gruppe „Pour un nouveau partenariat entre les organisations de solidarité internationale et les pouvoirs publics“, unter dem Vorsitz von Jean-Claude Faure.

Commission Coopération-Développement. Argent et organisations de solidarité internationale 20002001 (Résultats de l'enquête de la Commission Coopération Développement sur les ressources et dépenses des organisations de solidarité internationale en 2000 et 2001. Mise en perspective des chiffres de 1991 à 2001), Dezember 2003, Paris, Commission Coopération-Développement, 32 S.

DDC (Direction du développement et de la coopération), Aide de la Suisse aux pays en développement et aux pays en transition, Statistiques 2002, Bern, DDC, 2004, $200 \mathrm{~S}$.

Deler, J.-P., Fauré, Y.-A., Piveteau, A., Roca P.-J., ONG et développement. Société, économie, politique, Collection Hommes et sociétés, Paris, Ed. Karthala, 1998, 684 S.

Freymond, Jean F. , Boyer, Brook, Les Organisations non gouvernementales et la politique extérieure de la Suisse, Rapport de synthèse, Genève, CASIN (Centre d'études pratiques de la négociation internationale), 1998, $22 \mathrm{~S}$.

45 Venro, Verband Entwicklungspolitik Deutscher Nichtregierungsorganisationen, <www.venro.org > 
Gerster, Richard, „Die Zusammenarbeit zwischen dem Staat und privaten Hilfswerken. Erfahrungen aus der schweizerischen Entwicklungspolitik“ in Helmich, Henny; Bittner, Gerhard, (Hrsg.), Der dritte Sektor in der Entwicklungspolitik. Zusammenarbeit zwischen Staat und Nicht-Regierungsorganisationen NGOs. Wien, Österreichische Forschungsstiftung für Entwicklungshilfe, 1996, S. 135-145.

Helmich, Henny, Nouveaux partenariats pour la coopération au développement: orientations des années 1980 et défis des années 1990, Centre de développement, Paris, OCDE, 1990, 39 S.

Holenstein, René, Was kümmert uns die Dritte Welt. Zur Geschichte der internationalen Solidarität in der Schweiz, Zürich, Chronos Verlag, 1998, 264 S.

iuéd, DDC, Aide suisse aux pays en développement et aux pays en transition 1998-2000, Genf, iuéd, 2002, 206 S.

Landolf, Dani, Fankhauser, Michael, Gekaufte Entwicklungshilfe? Die (Un)Abhängigkeit der Nichtregierungsorganisationen von Geld und Einfluss der staatlichen Entwicklungspolitik, Köniz, Ed. Soziothek, Facharbeit Entwicklungssoziologie, 1996, 99 S.

Ministère des affaires étrangères, Organisations de solidarité internationale et pouvoirs publics en Europe. Etude comparative sur les dispositifs d'appui et de concertation. Note de synthèse, Paris, Direction générale de la coopération internationale et du développement, Juni 2001, 40 S.

Rosselet, Justine, ONG et management fondé sur la qualité: terre des hommes ou terre des normes?, Collection intinéraires Etudes du développement no 19, Genf, iuéd, September 2003, 76 S.

Ryfman, Philippe, Les ONG, Collection Repères, Paris, La Découverte, 2004, 124 S.

Smillie, Ian; Helmich, Henny (Hrsg.), Stakeholders. Government-NGO Partnerships for International Development, London, Earthscan Publications, OCDE, 1999, 318 S.

UNO Panel of Eminent Persons on Civil Society and UN Relationships : <www.un.org/reform/panel.htm>.

Wegner, Rodger, Nichtregierungsorganisationen und Entwicklungshilfe. Einführung und systematische Bibliographie, Hamburg, Schriften des Deutschen Übersee-Instituts Nr. 20, 1993, 147 S.

\section{Artikel}

Basler Zeitung, 14. Juli 2003, „Non-Profit-Organisationen suchen Zeit und Geld“ ; 14. November 2003, „Nonprofit-Organisationen wollen weisse Westen“.

Cash, 18. Mai 2001, „Das Kreuz mit den Millionen“.

Der Schweizerische Beobachter, Nr. 10, 14. Mai 1993, Dossier „Entwicklungshilfe: Vermarktung des Elends".

E+Z Entwicklung und Zusammenarbeit, Nr. 10, 1993 „Nichtregierungsorganisationen: Die Hoffnungsträger der 90er Jahre“, Nr. 11, November 1994 (Dossier Nichtregierungsorganisationen).

epd-Entwicklungspolitik, 5/1999, „Über den Vorteil, in der zweiten Reihe zu sitzen. Wirkungserfassung und Qualitätsmanagement bei einer Spendenorganisation“.

epd-Entwicklungspolitik, „Lobbyarbeit von Nichtregierungsorganisationen“, Tagungsdokumentation und Texte zur Rolle der NRO in der Entwicklungszusammenarbeit, Materalien IV/91, 1991, S. 9-91.

epd-Entwicklungspolitik, Mai 1999 (Dossier Nichtregierungsorganisationen).

Ghils, Paul, „La société civile internationale: les organisations internationales non gouvernementales dans le système international“", in: Revue internationale des sciences sociales, Nr. 133, August 1992, S. 467-481.

Glagow, Manfred, „Nichtregierungsorganisationen - und kein Ende“, in epd-Entwicklungspolitik 5/99, 1999, S. 23-27.

Handelszeitung, 9. Dezember 1998, „Die milden Gaben der Multis \& Co.“ ; 5. November 2003.

Hartmann, Stefan et ali, ,Spendenmarkt Schweiz“, in Mosquito Nr. 4, 1997, (Dossier) S. 4-23.

Helvetas, Partenaires, Dossier: Société civile et Etat, Nr. 176, Juni 2004.

L'Hebdo, 23. September 2004, Dossier „EEuvres d'entraide : le classement de 1'Hebdo“, 12 S.

Morales, Horacio M. et ali, „Die Nichtregierungsorganisationen des Südens. Von der Nothilfe zur Veränderung der Systeme“, in: E+Z Entwicklung und Zusammenarbeit, Nr. 10, Bonn, Deutsche Stiftung für internationale Entwicklung, 1993, S. 256-265.

Neue Zürcher Zeitung, 29. September 2000, „Von Seattle nach Prag. Der wachsende Einfluss der Nichtregierungsorganisationen“; 14. Juli 2003, „,Spenden für die Werbung um Spenden“.

Niggli, Peter, Rothenbühler André, „Haben die NGO ein Legitimitätsproblem ?“, in Global+, Nr. 4/2003.

Ryfman, Philippe, Organisations internationales et organisations non gouvernementales: partenaires, concurrents ou adversaires? Cahiers français Nr. 302, La Documentation française, Paris, 2001.

Der Schweizerische Beobachter, Nr.10, 14. Mai 1993, Dossier „Entwicklungshilfe: Vermarktung des Elends".

Tages-Anzeiger, 22. Juli 2003, „Ich verstehe den Ärger der Spendenden“ ; 23. Juli 2003 „Hilfswerke sammeln auf die kreative Tour". 


\section{ANHANG I}

\section{Zur privaten Hilfe der NRO in der Schweiz verfügbare Quellen}

Es ist unmöglich, vollständige Daten über die Entwicklungshilfe der NRO der Länder des Nordens zu erhalten. Wie im einleitenden Kapitel zum Dossier dargelegt, gibt es kein offizielles Verzeichnis, um alle nichtstaatlichen Organisationen zu erfassen, und es besteht keine Gesetzesvorschrift, welche die NRO zwingt, genaue Angaben zu ihrer Rechnungslegung zu machen. Es existiert somit keine Gesamtübersicht über die Finanzierung der NRO, und die partiellen Informationsquellen sind diverser Art. Es handelt sich dabei um Jahresberichte der NRO, um Jahresumfragen bei NRO, Kantonen und Gemeinden ${ }^{46}$, ferner um den NGO-Dienst und den Statistischen Diens ${ }^{47}$ der DEZA. Mehrere Organe veröffentlichen zudem regelmässig Listen von NRO, welche in bestimmten Bereichen tätig sind.

\section{Jahresumfragen zur Hilfe der Schweizer NRO, die auf der Grundlage der statistischen Leitlinien des OECD-Entwicklungshilfeausschusses DAC durchgeführt werden}

Von 1961 bis 1990 wurden von Swissaid und seit 1991 vom Genfer Universitätsinstitut für Entwicklungsstudien iuéd (im Auftrag der DEZA) Jahresumfragen durchgeführt, um die Entwicklungshilfe der NRO zu ermitteln, die durch deren Eigenmittel finanziert wird ${ }^{48}$. Anhand eines Adressenverzeichnisses von über tausend Institutionen „Schweiz-Dritte Welt” erhielten im Jahr 2003 mehr als 500 Hilfswerke Fragebögen, um die Liste der von ihnen im Jahr 2002 unterstützten Entwicklungshilfeprojekte anzugeben. Unter den 285 Organisationen, welche die Fragebögen beantworteten, gaben rund 250 an, im Jahr 2002 Projekte finanziert und verwaltet zu haben.

Die in der Schweiz durchgeführten Erhebungen beruhen auf den vier Kategorien von Finanzflüssen in die Entwicklungsländer, die vom Entwicklungshilfeausschuss der OECD (DAC) festgelegt wurden ${ }^{49}$ : 1) öffentliche Entwicklungshilfe (einschliesslich der öffentlichen Beiträge an die NRO); 2) andere Beiträge des öffentlichen Sektors; 3) Privatkapitalströme; 4) "Spenden der privaten freiwilligen Organisationen“. Letztere Kategorie umfasst die Leistungen von nicht gewinnorientierten Institutionen, ohne Subventionen des öffentlichen Sektors. Diese „Spenden der privaten freiwilligen Organisationen“ bilden den Gegenstand der Umfragen des iuéd.

Durch die DAC-Statistiken kann vermieden werden, gewisse Leistungen der NRO doppelt zu zählen, indem die Finanzflüsse zu dem Zeitpunkt eingetragen werden, zu dem sie in den Empfängerländern ausgezahlt werden. Die Angaben berücksichtigen zum Beispiel weder die Mittelüberweisung von einer NRO an eine andere, noch Überweisungen von einer nationalen Zweigstelle einer internationalen NRO an eine andere Zweigstelle der gleichen NRO, sondern sie registrieren die endgültige Zuteilung für die Finanzierung von Projekten vor Ort. Einige Organisationen, wie die Glückskette oder Brot für alle, führen Sammlungen in der Öffentlichkeit durch, verwalten aber selbst keine Projekte. Sie überweisen die Mittel an andere schweizerische Institutionen. Diese Beträge werden bei den NRO angegeben, welche die Projekte durchführen.

\section{In den privaten Hilfeflüssen sind enthalten:}

- Gelder, die im laufenden Jahr zur Finanzierung von Projekten der Entwicklungszusammenarbeit und von humanitären Einsätzen in den Entwicklungs- und Transitionsländern ausgezahlt werden. Dabei ist zu vermerken, dass die Daten bezüglich der Hilfe der NRO für ein Jahr die im laufenden Jahr effektiv

46 Die Umfragen bei Kantonen und Gemeinden liefern Informationen über die dezentralisierte Entwicklungszusammenarbeit, die in den meisten Fällen zur Finanzierung von NRO-Projekten dient.

47 Der Statistische Dienst trägt Angaben über die Beiträge von DEZA und seco an die NRO zusammen.

48 Die Ergebnisse der vom iuéd im Auftrag der DEZA durchgeführten Jahresumfragen wurden in der Broschüre: Schweizerische Hilfe für Entwicklungsländer und Oststaaten in jährlichen Ausgaben von 1991 bis 1998 veröffentlicht und 2002 unter dem Titel: Aide suisse aux pays en développement et aux pays en transition für die Jahre 1998 bis 2000 herausgebracht. Seit 2001 werden die Daten in der Broschüre der DEZA: Aide suisse aux pays en développement et aux pays en transition veröffentlicht.

49 Die OECD-Statistiken werden im Statistischen Anhang des Jahresberichts Les Dossiers du CAD: Coopération pour le développement, (Internetadresse: <www.oecd.org/dac >) veröffentlicht. 
ausgezahlten privaten Spenden angeben. Hingegen werden die über die NRO laufenden Hilfeleistungen der Kantone und Gemeinden sowie die Zahlungen der DEZA an die Hilfswerke in dem Jahr eingetragen, in dem die öffentliche Körperschaft den Betrag an die NRO auszahlt, und nicht bei der endgültigen Auszahlung ans Empfängerland. Somit können die Kantone und Gemeinden oder die DEZA Zahlungen an eine NRO am Ende eines Jahres leisten, und die betreffende NRO kann dieses Geld erst im folgenden Jahr für Projekte einsetzen.

口 Zahlungen an die UNICEF, welche im Rahmen von Sammelkampagnen des Schweizerischen Komitees für UNICEF geleistet werden; ferner Zahlungen an das Flüchtlingshilfswerk der Vereinten Nationen (UNHCR).

\section{Von den Statistiken nicht erfasste Hilfeflüsse}

Man muss sich der Tatsache bewusst sein, dass diese in der Schweiz verfügbaren Zahlenangaben nicht die gesamte von der Schweiz in die Entwicklungsländer transferierte Hilfe umfassen, und dies aus folgenden Gründen:

- Die Angaben basieren auf den Leistungen von 250 NRO, wogegen Adressenverzeichnisse 1000 bis 1500 Organisationen „Schweiz-Dritte Welt” umfassen. Wie bereits erwähnt, besteht keine gesetzliche Basis, um die NRO zu zwingen, die Angaben über ihre Mittelüberweisungen zu veröffentlichen, und die Daten beruhen auf den Informationen, welche die NRO anzugeben bereit sind.

口 Die in diesem Kapitel erwähnten Statistiken stammen aus verschiedenen bereits angemerkten Quellen (Jahresberichte der NRO, Auskünfte von Kantonen und Gemeinden, Statistischer Dienst der DEZA).

- Die durch die Jahresumfragen ermittelte Hilfe der NRO umfasst die im Jahr überwiesenen Beträge, welche durch die Eigenmittel der NRO finanziert werden (Sammlungen, Mitgliedsbeiträge oder Legate), ohne die öffentlichen Beiträge der DEZA bzw. der Kantone und Gemeinden, welche in der öffentlichen Entwicklungshilfe enthalten sind.

- Die Aufwendungen der NRO für die Aufnahme, Beratung und Unterstützung der Flüchtlinge in der Schweiz sind nicht in den Statistiken über die Hilfe der NRO enthalten.

ـ Ein Teil der Hilfe für kleine Projekte kann über Vereine für die Solidarität mit einem gegebenen Land oder über Vereinigungen von in der Schweiz wohnhaften Ausländern laufen, welche nicht unbedingt den alljährlichen Fragebogen erhalten.

- Die Statistiken erfassen die Angaben über die grossen NRO für Entwicklungszusammenarbeit ziemlich gut, jedoch ist ein Teil der direkten Hilfe schwieriger zu erfassen, wenn eine Person beispielsweise Geld an eine Bekanntschaft in einem Land des Südens überweist, ohne dass dies zwangsläufig über einen strukturierten Verein läuft.

• Die Zahlen berücksichtigen nicht die Mittelüberweisungen von in der Schweiz wohnhaften Ausländern an ihre Familie im Herkunftsland. Diese Überweisungen gehen jedoch weit über die Beträge der öffentlichen und privaten Hilfe hinaus. Gemäss der Weltbank überweisen die in der Schweiz wohnhaften Ausländer (aller Herkunftsländer) jährlich über 8 Milliarden Franken an ihre Familien.

\ Die Unterscheidung zwischen Missionshilfe einerseits und humanitärer Hilfe und Entwicklungszusammenarbeit andererseits ist bei den Kirchen nahe stehenden Institutionen nicht leicht vorzunehmen, da diese Hilfe für die genannten Organisationen oft ein Ganzes bildet.

口 Die DAC-Statistiken betreffen die Finanzflüsse in die Entwicklungs- und Transitionsländer und umfassen nicht die Aufwendungen in den Ländern des Nordens. Somit berücksichtigen die Umfragen nicht die Ausgaben der NRO in der Schweiz. Nicht enthalten sind die Ausgaben für die Sekretariate in der Schweiz, für Hilfsprojekte in der Schweiz oder in einem anderen Industrieland (z.B. Überschwemmungen in Italien), für Marketing oder für die Finanzierung von Sammelkampagnen in der Schweiz, ferner für die Bereiche Sensibilisierung, Öffentlichkeitsarbeit oder entwicklungspolitische Bildung in der Schweiz.

Quellen: DAC-Leitlinien zur Erstellung der Statistiken über die Finanzflüsse in die Entwicklungs- und Transitionsländer; Einführung zur Broschüre Aide suisse aux pays en développement et aux pays de l'Europe orientale 1998, iuéd, Genf, 1999, S. I-VII; Statistischer Dienst der DEZA. 


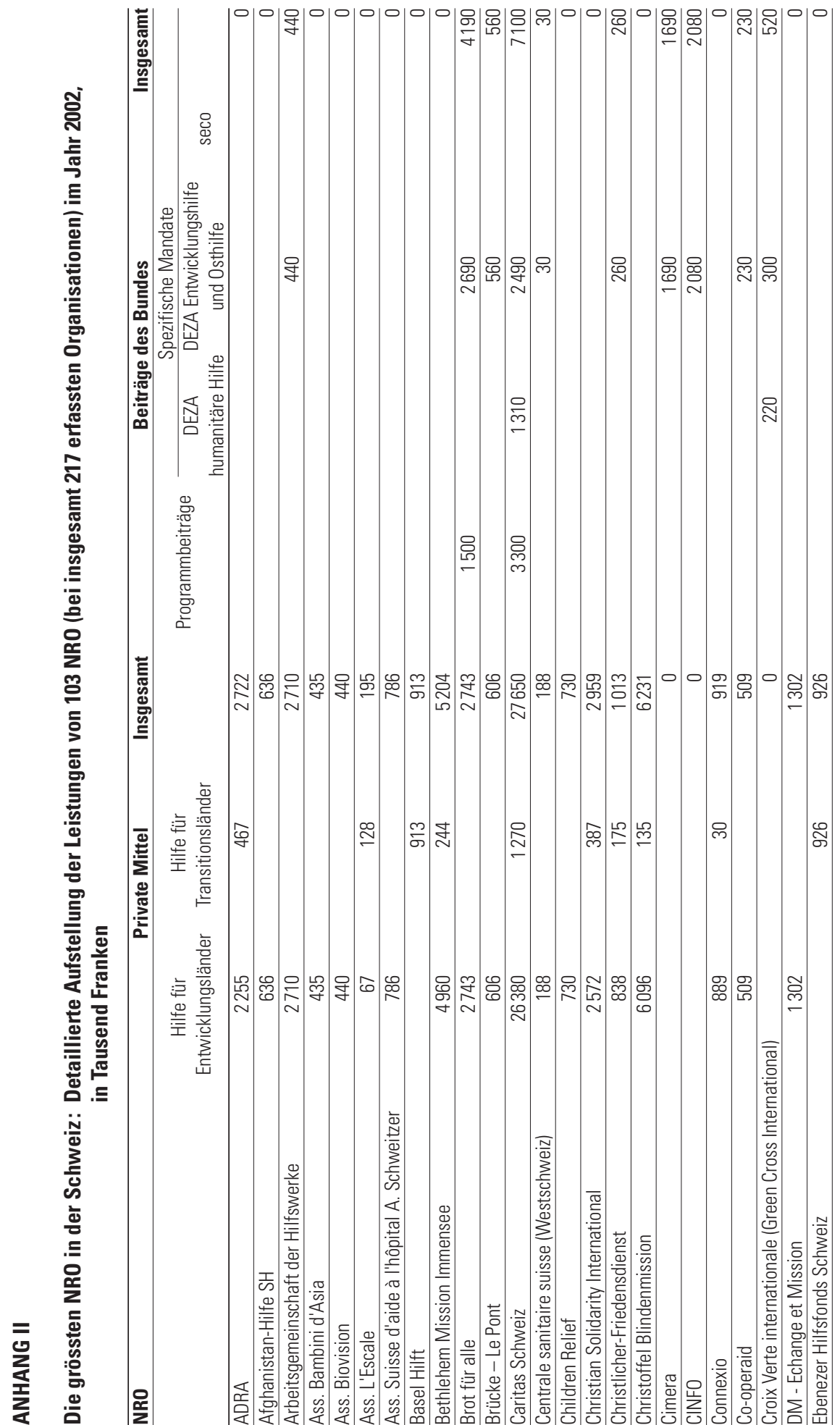




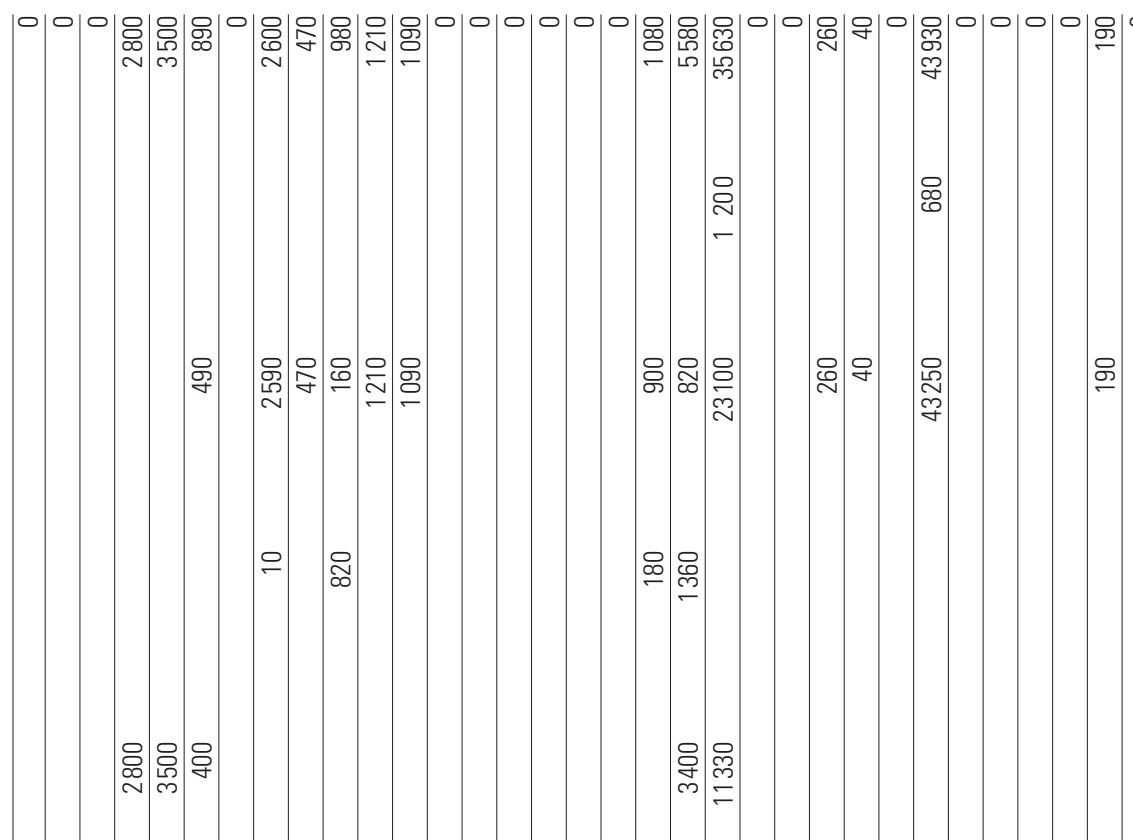

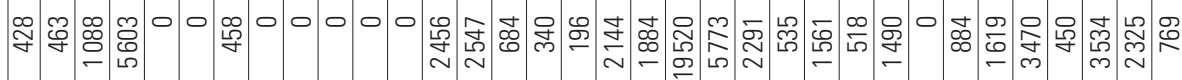

요

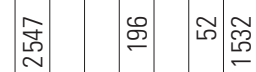

สิ

○)

क्?

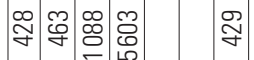

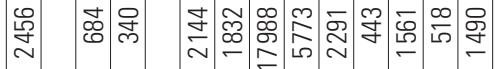

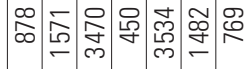

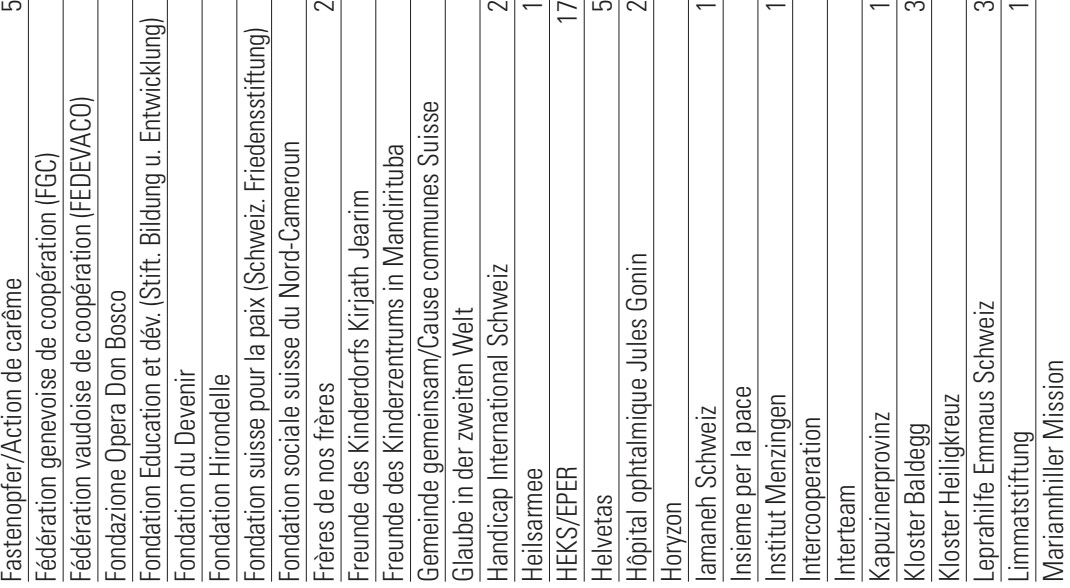


豆

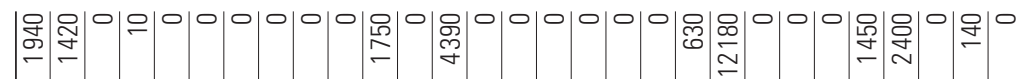

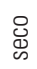

్ా

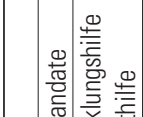

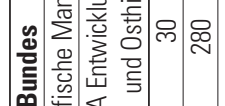

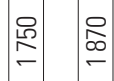

은 $\frac{0}{\sim}$

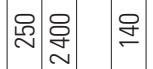

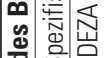

㐘

产

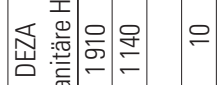

‡

요요유

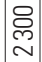

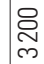

兽

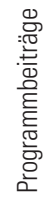

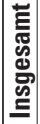

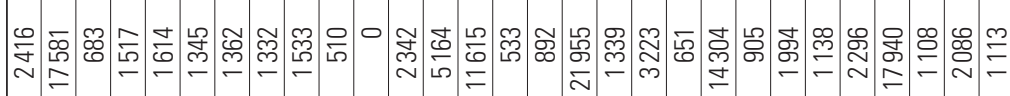

เ

|.

$\frac{\bar{\Phi}}{\mathrm{c}}$

ஜ

웅

8 学

유

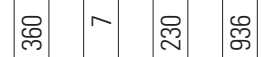

$\stackrel{\sim}{\sim}$

竞

离

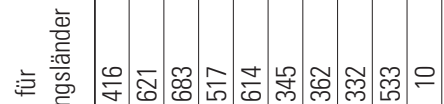

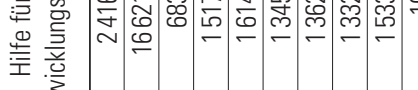
站

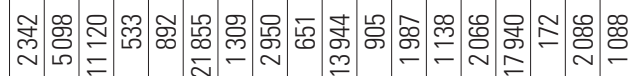
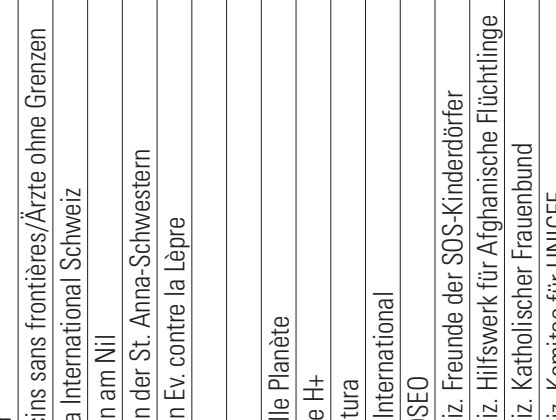

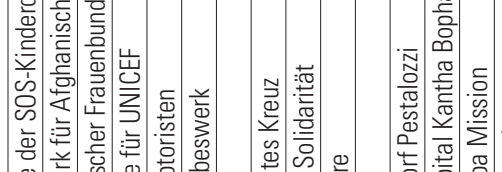

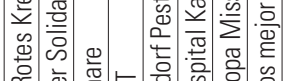

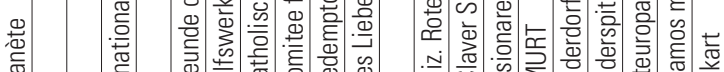

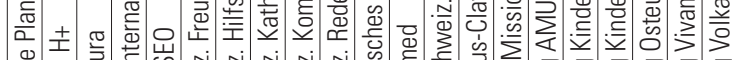

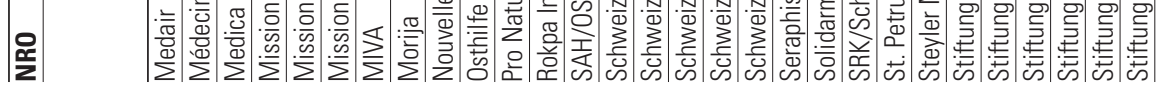




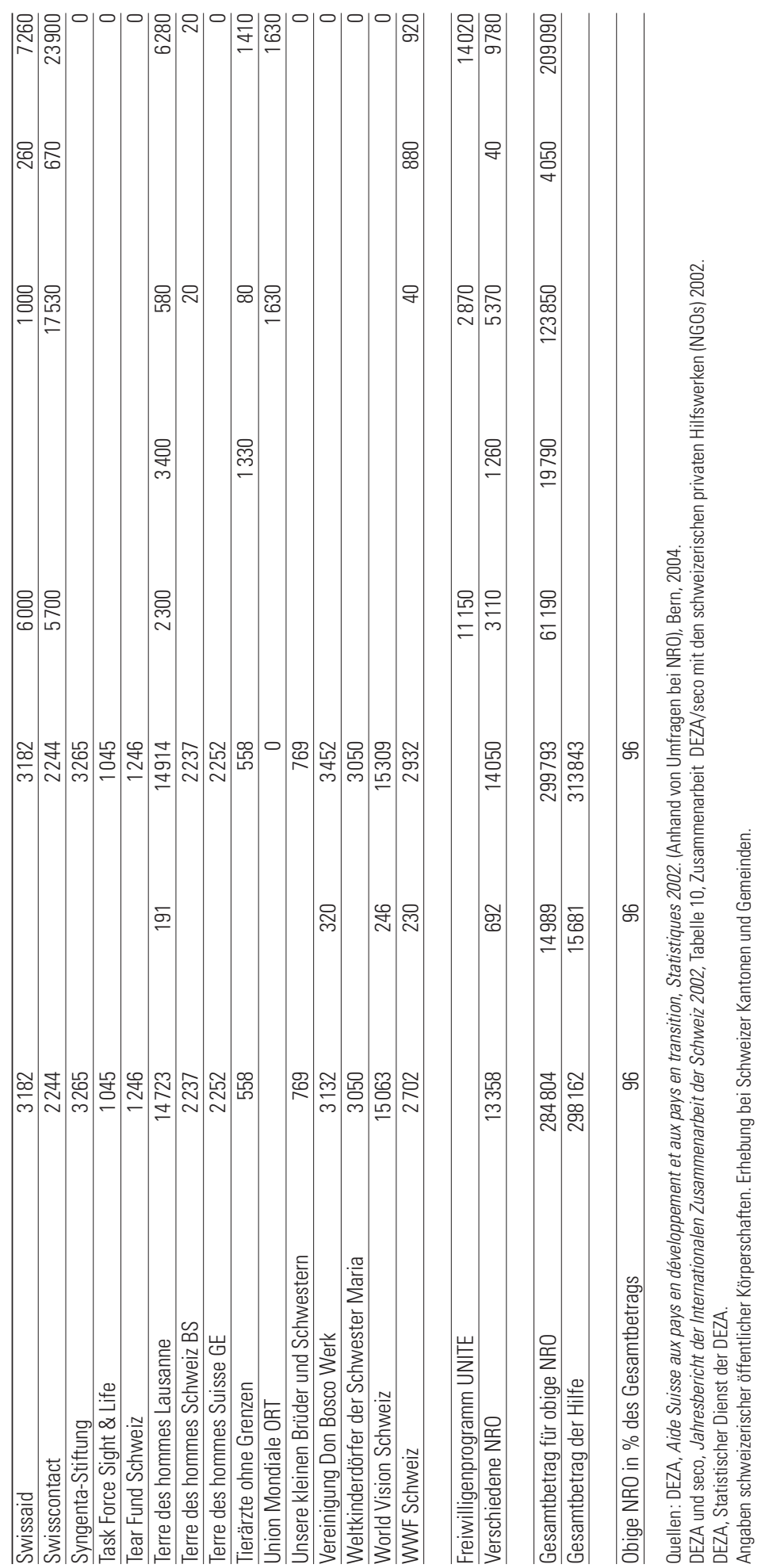




\section{ANHANG III}

Zusammensetzung der Einnahmen und Ausgaben einiger schweizerischer NRO im Jahr 2003

\begin{tabular}{lrr}
\hline Caritas Schweiz & in Tausend Franken & in \% des Gesamtbetrags \\
\hline Gesamterträge & 103120 & \\
\hline Erträge des Bereichs internationale Zusammenarbeit & 43047 & 100,0 \\
\hline davon: Spenden, Legate & 7895 & 18,3 \\
\hline Kantone und Gemeinden & 892 & 2,1 \\
\hline Beiträge des Bundes & 10919 & 25,4 \\
\hline Beiträge der Glückskette und verschiedene Beiträge & 23341 & 54,2 \\
\hline Gesamtausgaben & 103120 & 100,0 \\
\hline davon: Internationale Zusammenarbeit & 40285 & 39,1 \\
\hline Soziale Aktion in der Schweiz & 6707 & 6,5 \\
\hline Migration & 30320 & 29,4 \\
\hline Personalkosten (alle Bereiche) & 21939 & 21,3 \\
\hline Kommunikation & 1252 & 1,2 \\
\hline
\end{tabular}

\begin{tabular}{lrr}
\hline Helvetas & in Tausend Franken & in \% des Gesamtbetrags \\
\hline Gesamterträge & 53180 & 100,0 \\
\hline davon: Private Spenden und Legate & 6597 & 12,4 \\
\hline Lokale Sektionen & 1026 & 1,9 \\
\hline DEZA Programmbeiträge und Regieprojekte & 29388 & 55,3 \\
\hline Mandate & 13721 & 25,8 \\
\hline Kantone und Gemeinden & 794 & 1,5 \\
\hline Gesamtausgaben & 53180 & 100,0 \\
\hline davon: Ausgaben für Auslandsprojekte & 43790 & 82,3 \\
\hline Verwaltung, Sekretariate & 5000 & 9,4 \\
\hline Projekte in der Schweiz & 3385 & 6,4 \\
\hline
\end{tabular}

\begin{tabular}{lcr}
\hline Ärzt ohne Grenzen Schweiz & in Tausend Franken & in \% des Gesamtbetrags \\
\hline Gesamterträge & 52570 & 100,0 \\
\hline davon: In der Schweiz aufgebrachte private Spenden & 34175 & 65,0 \\
\hline Private Spenden anderer Sektionen & 10204 & 19,4 \\
\hline Institutionelle Einnahmen & 7593 & 14,4 \\
\hline Gesamtausgaben & 49712 & 100,0 \\
\hline davon: Kosten der Projekte vor Ort & 35752 & 71,9 \\
\hline Unterstützung der Projekte vom Sitz aus & 5272 & 10,6 \\
\hline Sensibilisierung & 709 & 1,4 \\
\hline Information und Fundraising & 4938 & 9,9 \\
\hline Verwaltung & 3041 & 6,1 \\
\hline
\end{tabular}

\begin{tabular}{lrr}
\hline Swisscontact & in Tausend Franken & in \% des Gesamtbetrags \\
\hline Gesamterträge & 34974 & 100,0 \\
\hline davon: Private Spenden & 1643 & 4,7 \\
\hline Beiträge von Kantonen und Gemeinden & 904 & 2,6 \\
\hline Bund, Regieprojekte/Mandate & 18889 & 54,0 \\
\hline Bund, projektgebundene Beiträge & 4860 & 13,9 \\
\hline Andere Bundesbeiträge & 3868 & 11,1 \\
\hline Gesamtausgaben & 34974 & 100,0 \\
\hline davon: Projekte & 29958 & 85,7 \\
\hline Projektbegleitung und Verwaltung & 3547 & 10,1 \\
\hline Information und Fundraising & 335 & 1,0 \\
\hline
\end{tabular}




\begin{tabular}{lrr}
\hline Stiftung Terre des Hommes Lausanne & in Tausend Franken & in \% des Gesamtbetrags \\
\hline Gesamterträge & 33234 & 100,0 \\
\hline davon: Partnerschaften, Spenden, Legate & 18451 & 55,5 \\
\hline Glückskette & 1226 & 3,7 \\
\hline Mäzenatentum und Unternehmen, Stiftungen & 1628 & 4,9 \\
\hline Bund & 5356 & 16,1 \\
\hline Kantone und Gemeinden & 459 & 1,4 \\
\hline Einnahmen im Ausland & 3234 & 9,7 \\
\hline Gesamtausgaben & 32131 & 100,0 \\
\hline davon: Programme im Ausland & 22888 & 71,2 \\
\hline Programme in der Schweiz & 1763 & 5,5 \\
\hline Information in der Schweiz & 288 & 0,9 \\
\hline Fundraising und damit verbundene Kommunikation & 3596 & 11,2 \\
\hline Allgemeine Verwaltung & 2585 & 8,0 \\
\hline & & \\
\hline World Vision. Jahr 2002/2003 & in Tausend Franken & in \% des Gesamtbetrags \\
\hline Gesamterträge & 26877 & 100,0 \\
\hline davon: Partnerschaften & 21487 & 79,9 \\
\hline Öffentliche Beiträge & 920 & 3,4 \\
\hline WFP & 1107 & 4,1 \\
\hline Gesamtausgaben & 26877 & 100,0 \\
\hline davon: Projekte & 17564 & 65,3 \\
\hline Verwaltung & 3401 & 12,7 \\
\hline Information/Sammelspesen & 1707 & 6,4 \\
\hline
\end{tabular}

\begin{tabular}{lcr}
\hline Swissaid & in Tausend Franken & in \% des Gesamtbetrags \\
\hline Gesamterträge & 14474 & 100,0 \\
\hline davon: Spenden & 4461 & 30,8 \\
\hline Anzeichenverkauf & 1485 & 10,3 \\
\hline Glückskette & 665 & 4,6 \\
\hline Subventionen des Bundes & 6084 & 42,0 \\
\hline Gesamtausgaben & 14054 & 100,0 \\
\hline davon: Projekte im Ausland & 9290 & 66,1 \\
\hline Projekte in der Schweiz & 794 & 5,6 \\
\hline Personalkosten & 2006 & 14,3 \\
\hline Verwaltungskosten & 569 & 4,0 \\
\hline Information & 446 & 3,2 \\
\hline Fundraising & 836 & 5,9 \\
\hline Christoffel-Blindenmission & & \\
\hline Gesamterträge & in Tausend Franken & in \% des Gesamtbetrags \\
\hline davon: Spenden & 6952 & 100,0 \\
\hline Legate & 5326 & 76,6 \\
\hline Gesamtausgaben & 1177 & 16,9 \\
\hline davon: Projekte & 6952 & 100,0 \\
\hline Bildung/Öffentlichkeitsarbeit & 4489 & 64,6 \\
\hline Verwaltung & 1617 & 23,3 \\
\hline Tierärzte ohne Grenzen Schweiz & 807 & 11,6 \\
\hline Gesamterträge & & \\
\hline davon: Bund - DEZA & in Tausend Franken & in \% des Gesamtbetrags \\
\hline Private Spenden & 4240 & 100,0 \\
\hline Europäische Union - ECHO und Somalia Unit & 1121 & 26,4 \\
\hline Gesamtausgaben & 121 & 2,8 \\
\hline davon: Projektkosten & 2243 & 52,9 \\
\hline Sitzkosten & 4241 & 100,0 \\
\hline & 4138 & 2,4 \\
\hline
\end{tabular}


Quellen: Jahresberichte 2003 der verschiedenen NRO

Anmerkungen: Die Rechnungslegung ist in den verschiedenen NRO nicht vereinheitlicht.

Die Fundraisingkosten sind selten getrennt identifizierbar. Sie sind zumeist in anderen Rubriken enthalten. Die Definition der Verwaltungskosten in der Schweiz kann von einer Organisation zur anderen unterschiedlich sein.

Die Beiträge der Kantone und Gemeinden sind oft in den privaten Spenden eingeschlossen. 